Aus der Abteilung Allgemeinmedizin

(Prof. Dr. med. M. M. Kochen, MPH, FRCGP)

im Zentrum Innere Medizin

der Medizinischen Fakultät der Universität Göttingen

\title{
Interdisziplinäre Kommunikation bei der Überweisung von Patienten mit Kreuzschmerzen vom Hausarzt zum Orthopäden
}

\author{
INAUGURAL - DISSERTATION \\ zur Erlangung des Doktorgrades \\ der Medizinischen Fakultät
}

der Georg-August-Universität zu Göttingen

vorgelegt von
Anna Pieper
aus
Braunschweig

Göttingen 2009 
Dek a n:

I. Berichterstatter:

II. Berichterstatter/in:

III. Berichterstatter/in:
Prof. Dr. med. C. Frömmel

PD Dr. med. J.-F. Chenot

Tag der mündlichen Prüfung: 


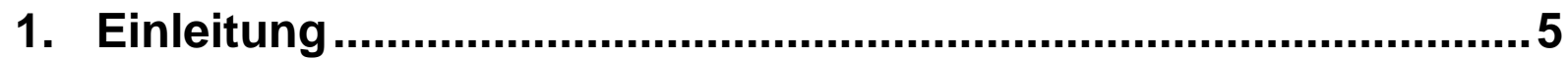

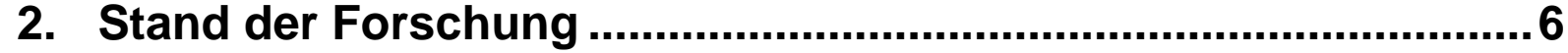

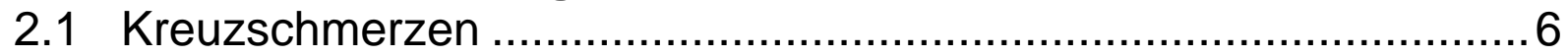

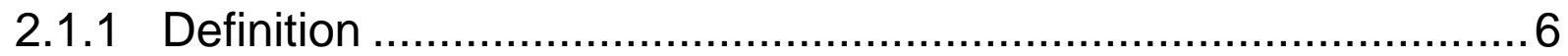

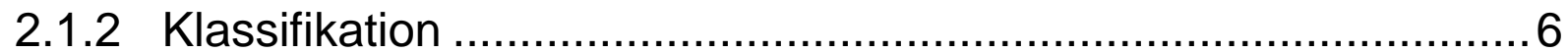

2.1.3 Epidemiologie ............................................................ 7

2.1.4 Hausärztliche Diagnostik und Therapie................................. 8

2.2 Strukturelle Rahmenbedingungen des Gesundheitssystems...........9

2.3 Überweisung von Kreuzschmerzpatienten zum Orthopäden ......... 10

3. Fragestellung und Zielsetzung ....................................................13

4. Material und Methoden ............................................................... 14

4.1 Studiendesign................................................................... 14

4.2 Methodische Vorüberlegungen ........................................... 14

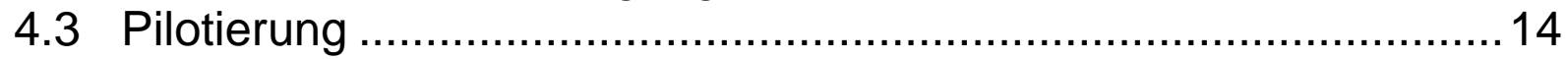

4.4 Auswahlverfahren der Praxen ............................................... 15

4.5 Rekrutierung der Hausärzte ................................................. 15

4.6 Datenerhebung in der Praxis ................................................ 15

4.7 Arztfragebögen.................................................................. 16

4.8 Inhaltliche Analyse der Arztbriefe ......................................... 17

4.9 Ethik und Datenschutz ................................................... 18

4.10 Auswertung ................................................................ 18

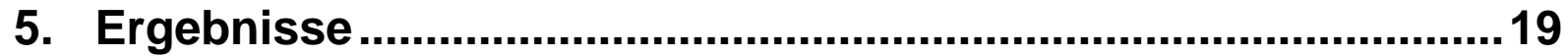

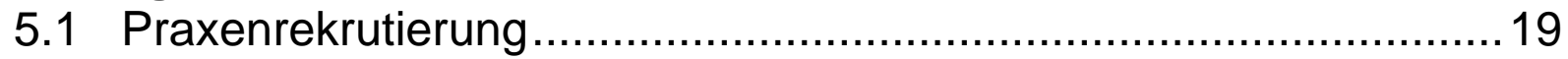

5.2 Beschreibung der Praxen ........................................................ 20

5.3 Beschreibung der gefundenen Überweisungen/Patienten ............20

5.4 Selbsteinschätzung der Hausärzte ...........................................21

5.5 Angaben der Hausärzte auf dem Überweisungsschein ................22

5.6 Patientencharakteristika und Gründe für die Überweisung ............23

5.7 Auswertung der orthopädischen Arztbriefe ...............................24

5.8 Bewertung der orthopädischen Arztbriefe durch die Hausärzte .....26

6. Diskussion ................................................................................28

6.1 Zusammenfassung der wichtigsten Ergebnisse ........................28

6.2 Stärken und Schwächen dieser Doktorarbeit .............................28

6.3 Kritische Betrachtung der Ergebnisse .....................................29

6.4 Vergleich mit der internationalen Literatur..................................32

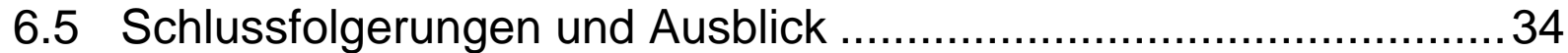

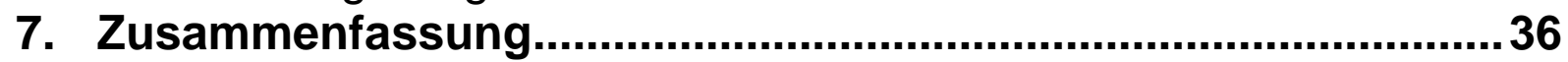

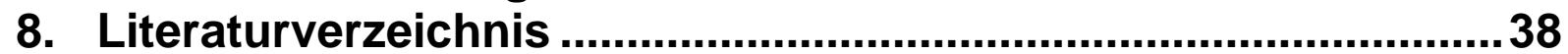

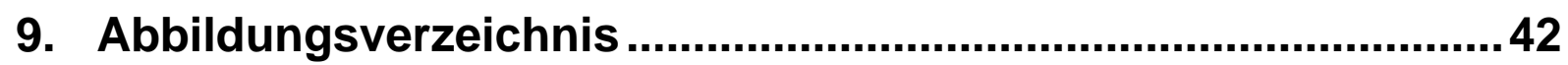

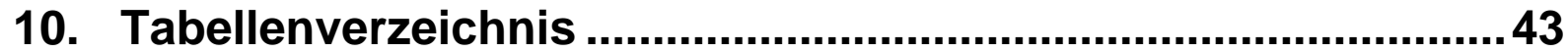

11. Abkürzungsverzeichnis ..................................................... 44

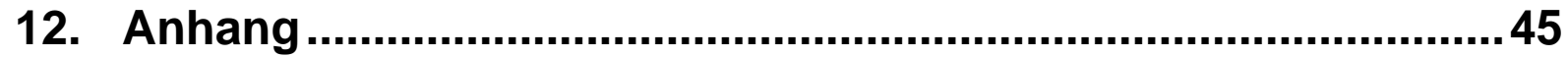




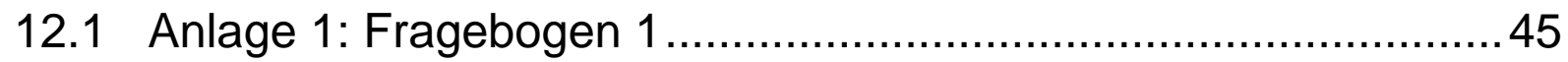

12.2 Anlage 2: Fragebogen 2 .................................................. 46

12.3 Anlage 3: Procedure Normal ................................................. 47

12.4 Anlage 4: Erstdokumentation Rückenschmerzen .....................49

12.5 Anlage 5: Votum Ethikkommission .......................................51

12.6 Anlage 6: Patientenbegleitdokumentation AOK ......................52 


\section{Einleitung}

Kreuzschmerzen sind ein epidemiologisch und ökonomisch relevantes Krankheitsbild. Der guten Prognose mit hoher Spontanheilungsrate für die Mehrheit der betroffenen Patienten steht ein chronischer Verlauf mit langen Arbeitsausfällen bei einem Teil der Patienten gegenüber. Die primäre Versorgung von Kreuzschmerzpatienten erfolgt durch Hausärzte. Eine Überweisung zum Spezialisten ist indiziert, wenn diagnostische oder therapeutische Möglichkeiten des Hausarztes nicht ausreichen. Bisher existiert hierfür keine klare Schnittstellendefinition. Hinweise auf einen abwendbar gefährlichen Verlauf (sog. „red flags“) oder persistierende Beschwerden ohne Besserungstendenz gelten als Überweisungsindikation.

Überweisungen zum Orthopäden sind häufig. In hausärztlichen Praxen in Deutschland wird jeder fünfte Patient, der eine Überweisung erhält, zum Orthopäden überwiesen (Rosemann et al. 2006). Für eine gute Versorgung der Patienten ist eine effiziente Kommunikation zwischen Hausärzten und Orthopäden wünschenswert. 


\section{Stand der Forschung}

\subsection{Kreuzschmerzen}

\subsubsection{Definition}

Der umgangssprachlich verwendete Begriff „Rückenschmerzen“ ist unscharf begrenzt. Unter dieser Bezeichnung sammeln sich Nackenschmerzen, Schmerzen im Brust- und Lendenwirbelbereich. Deshalb wird in Leitlinien meist der Begriff Kreuzschmerzen verwendet (Deutsche Gesellschaft für Allgemeinmedizin und Familienmedizin, Leitlinie 3 Kreuzschmerzen (DEGAM 2003)).

In der ICD-10 sind Rückenschmerzen unter M 54.5 definiert als „Schmerzen oder Unwohlsein im Bereich des Rückens vom unteren Rippenbogen bis zu den Glutäalfalten, eventuell mit Ausstrahlung in die Beine, die zu Einschränkungen bei den täglichen Verrichtungen führen." (DEGAM 2003). Zeitlich wird zwischen akuten, subakuten, chronischen und rezidivierenden Kreuzschmerzen unterschieden. Als akut bezeichnet man Schmerzen, die weniger als 12 Wochen bestehen. Subakut sind Schmerzen ab einer Dauer von 6 bis 12 Wochen. Von chronischen Kreuzschmerzen spricht man ab einer Dauer von mindestens 12 Wochen. Die Beschwerden können in ihrer Intensität und Ausprägung variieren. Nach einem schmerzfreien Intervall von 6 Monaten spricht man von rezidivierenden Kreuzschmerzen und behandelt diese wie eine erneute Episode akuter Kreuzschmerzen (Von Korff 1994).

\subsubsection{Klassifikation}

Ätiologische Klassifikationsversuche von Kreuzschmerzen sind bisher gescheitert, da es auch mit aufwendiger Diagnostik bei der Mehrheit der Patienten nicht gelingt, die Ursache der Beschwerden sicher festzustellen (Müller 2001). Daher haben sich international gültige und pragmatisch an der Prognose orientierte Klassifikationen durchgesetzt (Waddell 1982).

Die Mehrheit der hausärztlichen Patienten (> 90\%) fällt in die Gruppe unkomplizierter beziehungsweise unspezifischer Kreuzschmerzen und ist innerhalb von 1-2 Wochen schmerzfrei bzw. wieder arbeitsfähig (Pengel et al. 2003). Die Begriffe „unkompliziert“ und "unspezifisch“ sind ähnlich, aber sie unterscheiden sich dennoch im Denkansatz. 
„Unkompliziert" bezieht sich auf die gute Prognose, während „unspezifisch“ bedeutet, dass keine ätiologisch und klinisch sicher relevante Pathologie nachweisbar ist.

Ein Großteil der Patienten ist in gutem Allgemeinzustand und zeigt keine Lähmungen oder sensiblen Ausfälle. Die Schmerzen sind bewegungsabhängig und können bis oberhalb des Knies ausstrahlen (sogenannte „pseudo-radikuläre Schmerzen“). Nur in ca. $5 \%$ der Kreuzschmerzen im hausärztlichen Bereich liegt eine Nervenwurzelreizung vor. Die Schmerzen strahlen dann meist segmental bis unterhalb des Knies aus, wobei der Beinschmerz oft stärker als der Kreuzschmerz empfunden werden kann. Der Lasègue-Test ist positiv und es können Reflexauffälligkeiten beobachtet werden. Die Gefahr der Chronifizierung ist bei radikulären Kreuzschmerzen hoch. Lediglich ca. 1\% bildet die Gruppe der sogenannten komplizierten Kreuzschmerzen, denen eine relevante spezifische Pathologie wie z.B. Metastasen, Frakturen, Infektionen oder entzündliche Prozesse zu Grunde liegen (Gesundheitsberichterstattung, Deyo 2001).

\subsubsection{Epidemiologie}

Kreuzschmerzen sind eine universelle Erfahrung. Nahezu jeder Mensch leidet mindestens einmal im Laufe seines Lebens darunter. Zu jedem beliebigen Zeitpunkt (Punktprävalenz) geben bis zu 40\% der Bevölkerung an, Kreuzschmerzen zu haben. Die 1-Jahres-Prävalenz überschreitet $70 \%$ und die Lebenszeitprävalenz sogar $85 \%$ (Schmidt et al. 2007). Eine stetige Zunahme der Punktprävalenz von Kreuzschmerzen wird seit den siebziger Jahren beobachtet (Raspe und Kohlmann 1993).

Auch wenn die Mehrheit der Betroffenen keine medizinische Hilfe in Anspruch nimmt, gehören Kreuzschmerzen zu den gesundheitsökonomisch und volkswirtschaftlich bedeutsamen Erkrankungen (Göbel 2001). Sie spielen in nahezu allen Leistungsstatistiken des deutschen Gesundheitswesens eine dominante Rolle (Bolten et al. 1998, Krauth et al. 2005).

Kreuzschmerzen sind nach den Abrechnungsstatistiken der Krankenkassen der dritthäufigste Konsultationsanlass in der Hausarztpraxis (20\% aller Konsultationsabrechnungen) und der häufigste Konsultationsanlass bei ambulanten Orthopäden (50\% aller Konsultationsabrechungen) (Kassenärztliche Vereinigung Nordrhein 2006). 
Im Durchschnitt machen Krankheiten der Wirbelsäule und des Rückens ca. 15\% der gesamten Arbeitsunfähigkeitstage aus (Gesundheitsberichterstattung des Bundes). Die direkten Kosten wie Arzneimittelkosten, ambulante, stationär kurative und rehabilitative Behandlungsaufwendungen und die indirekten Kosten wie Produktionsausfälle durch Arbeitsunfähigkeitstage und vorzeitige Verrentung wurden 1999 auf etwa 50 Milliarden DM veranschlagt (Gesundheitsberichterstattung).

\subsubsection{Hausärztliche Diagnostik und Therapie}

Eine ausführliche Anamnese und körperliche Untersuchung bilden die Grundlage für die Entscheidung, ob weitere technische Untersuchungen oder eine Überweisung zum Spezialisten notwendig sind. Die Anamnese umfasst Fragen nach der Schmerzlokalisation, -dauer und -ausstrahlung, einem auslösenden Ereignis sowie nach Begleitsymptomen. Die klinische Untersuchung besteht aus Inspektion, Palpation sowie der Überprüfung der Kraft und Sensibilität. Die einzelnen Untersuchungen haben eine geringe Sensitivität und Spezifität. Durch Kombination mehrerer Untersuchungen kann ein gefährlich abwendbarer Verlauf im Regelfall ausgeschlossen werden (Chenot et al. 2006).

Bildgebende Untersuchungen sollten nicht routinemäßig, sondern nur bei Verdacht auf eine spezifische oder radikuläre Pathologie erfolgen. Bei Patienten unter 50 Jahren ohne Hinweis auf eine systemische Erkrankung wird durch radiologische Untersuchungen keine Behandlungsverbesserung erzielt (Kendrick et al. 2001). In der Mehrheit der Fälle wird keine zugrundeliegende Pathologie erkennbar sein.

Da Kreuzschmerzen eine hohe Spontanheilungsrate von ca. 90\% innerhalb von sechs Wochen haben, ist unnötige weiterführende Diagnostik und damit verbundenes verstärktes Krankheitserleben zu vermeiden. Daher ist die Aufklärung und Beratung des Patienten, dass es sich im Normalfall um keine ernsthafte Erkrankung handelt und dass eine schnelle Besserung zu erwarten ist, ein wichtiger Therapiebaustein (DEGAM 2003). Die beste Behandlungsoption ist die Aktivierung zu Bewegung und Wiederaufnahme der Arbeit. Routinemäßig sollen daher bei akuten, unkomplizierten Kreuzschmerzen keine passiven Behandlungsmodalitäten, wie z.B. Massage, verschrieben werden, da sie das Krankheitserleben und -verhalten verstärken. Damit Bewegung schmerzfrei möglich wird, empfiehlt sich eine konsequente Schmerztherapie. In erster Linie wird Paracetamol empfohlen und bei unzureichender Wirkung einfache nichtsteroidale Antirheumatika (NSAR). 
Muskelrelaxanzien werden, wenn überhaupt, nur kurzfristig allein oder in Kombination mit NSAR empfohlen.

Die Therapie der rezidivierenden und chronischen Kreuzschmerzen ist für Patienten und Therapeuten oft unbefriedigend. Sie erfordert eine regelmäßige Reevaluation des Patienten, ob tatsächlich keine Hinweise auf komplizierte Kreuzschmerzen vorliegen und ob daher eine Intensivierung der Therapie angezeigt ist. Die Betreuung wird in Kooperation mit Spezialisten, z.B. Orthopäden und Psychotherapeuten, durchgeführt.

Kann mit der Therapie das gemeinsame Ziel Schmerzfreiheit nicht erreicht werden, liegt der neue Schwerpunkt auf Verbesserung und Erhalt der Beweglichkeit. Die Motivation zu körperlicher Aktivität, die eine weitere Dekonditionierung vermeiden soll, stellt eine schwierige Herausforderung dar. Physiotherapie und Rückenschulungen, wie sie beispielsweise von Krankenkassen angeboten werden, können zur Aktivierung der Patienten genutzt werden. Mit zunehmender Dauer der Arbeitsunfähigkeit wird eine Rückkehr in das Arbeitsleben immer unwahrscheinlicher. Nach sechs Monaten kehren weniger als die Hälfte der Patienten in den Arbeitsprozess zurück. Frühzeitig sollten hier sogenannte multidisziplinäre Behandlungskonzepte, die unter anderem Schmerz- und Physiotherapie mit verhaltenstherapeutischen Verfahren kombinieren, in Erwägung gezogen werden. In ihrer Effektivität sind sie anderen Therapien überlegen (Pfingsten und Hildebrandt 2001). Entsprechende Angebote sind in Deutschland selten; deren Ziele können meist nur im Rahmen einer ambulanten oder stationären Rehabilitation verwirklicht werden. Bei längerer Arbeitsunfähigkeit aufgrund von Kreuzschmerzen sollte eine Rehabilitation der Patienten eingeleitet werden.

\subsection{Strukturelle Rahmenbedingungen des Gesundheitssystems}

Die Trennung des Gesundheitssystems in verschiedene Versorgungsebenen ermöglicht Arbeitsteilung, höhere Spezialisierung und die Bildung gut organisierter Funktionsbereiche. Ein Nachteil besteht in mangelndem zeitlichen und sachlichen Ineinandergreifen der einzelnen Behandlungen (Sachverständigenrat zur Begutachtung der Entwicklung im Gesundheitswesen 2001). Strukturierend werden Schnittstellendefinitionen, also klare Indikationen zur Mit- oder Weiterbehandlung 
durch bestimmte Spezialisten benötigt, die im deutschen Gesundheitssystem nicht verbindlich definiert sind (Hensler und Jork 2002).

Es gibt in Deutschland kein Primärarzt-System. Die Kassenpatienten haben unter den zugelassenen Vertragsärzten freie Wahl; der Zugang zu Spezialisten wird nicht durch den Hausarzt gesteuert. In anderen Gesundheitssystemen, wie z.B. in den Niederlanden, sind Hausärzte primäre Ansprechpartner für alle Gesundheitsprobleme, die den Zugang zu Spezialisten steuern (Knottnerus und ten Velden 2007).

Die Lotsenfunktion („gatekeeping“) des Hausarztes sollte mit der Einführung der Praxisgebühr im Jahre 2004 gestärkt werden. Patienten, die ohne eine Überweisung ihres Hausarztes einen Spezialisten aufsuchen, zahlen seitdem 10,-Euro/Quartal Praxisgebühr beim Spezialisten. Bei einer Überweisung durch den Hausarzt wird die Praxisgebühr nur einmalig beim Hausarzt fällig. Der Anteil der überwiesenen Patienten durch die Hausärzte ist seit der Einführung der Praxisgebühr in Niedersachsen von 23\% im Jahre 2003, auf 46\% 2004 gestiegen (Merten 2008).

Patienten erhalten jedoch auch ohne direkten Arztkontakt eine Überweisung beim Hausarzt, da es weder strukturelle Voraussetzungen, wie beispielsweise ein Listensystem, noch finanzielle Anreize für die Koordination durch den Hausarzt gibt. Die Steuerungsfunktion durch die Praxisgebühr ist in den hausärztlichen Praxen nicht eingetreten.

\section{3 Überweisung von Kreuzschmerzpatienten zum Orthopäden}

Die meisten Überweisungen bei Kreuzschmerzen gehen an niedergelassene Orthopäden, andere Spezialisten spielen nur eine untergeordnete Rolle (Chenot et al. 2008). Es gibt keinen anerkannten Konsens zwischen Hausärzten und Orthopäden darüber, wann welche Patienten überwiesen werden sollen. Bei Kreuzschmerzen werden die sogenannten „red flags“ als Warnhinweise auf komplizierte Kreuzschmerzen (siehe Tabelle 1) als Indikation zur Überweisung angesehen. Diese „red flags“ sind schlecht validiert und führen in der Praxis zur starken Überschätzung des Anteils komplizierter Kreuzschmerzen. Die DEGAMLeitlinie für Kreuzschmerzen empfiehlt eine Überweisung bei anhaltenden oder therapieresistenten Kreuzschmerzen ohne genaue Zeitangabe (DEGAM 2003). Bei 
schweren neurologischen Ausfällen oder schwerem Trauma wird die Krankenhauseinweisung empfohlen. Eine interdisziplinäre Arbeitsgruppe der Bertelsmannstiftung zum Qualitätsmanagement im Versorgungsprozess von Kreuzschmerzpatienten hat die „red flags“ in „dark red flags“ (sehr dringlich) und in „orange flags“ (weniger dringlich) eingeteilt (Bertelsmann-Stiftung 2007). „Dark red flags“ stellen z.B. die Lähmungssyndrome mit sofortigem Handlungsbedarf durch Spezialisten dar, die in der ambulanten Praxis eher selten vorkommen. Es wird auch vorgeschlagen, Patienten mit Krankschreibung innerhalb von zwei Wochen und Patienten ohne Krankschreibung innerhalb von 4 Wochen an einen Spezialisten zu überweisen, wenn keine deutliche Besserung der Symptomatik eintritt. In diesem Zeitraum sind bei ca. 80\% der Patienten Schmerz und Funktion deutlich gebessert (Pengel et al. 2003).

- $\quad$ Alter des Patienten <20 oder >55 Jahre

- $\quad$ kürzlich erlittenes adäquates Trauma

- reduzierter Allgemeinzustand / unklarer Gewichtsverlust

- Fieber

- Drogenmissbrauch, Immunsuppression, HIV

- Hinweis auf tumoröse oder rheumatisch - entzündliche Erkrankungen

- $\quad$ systemische Langzeitsteroidtherapie

- $\quad$ ausgeprägte neurologische Ausfälle / Cauda-Equina-Syndrom (Reithosenanästhesie, Mastdarm- \& Blasenlähmungen)

- $\quad$ strukturelle Deformitäten

Tabelle 1: Warnhinweise auf komplizierte Kreuzschmerzen („red flags“)

Die Kommunikation zwischen Hausärzten und Orthopäden findet weitgehend schriftlich statt. Die Überweisung erfolgt auf einem Vordruck, der von der Kassenärztlichen Bundesvereinigung (Muster 6) erstellt wurde. Neben den Patientendaten kann der Hausarzt einen kurzen Überweisungstext hinzufügen. Dazu stehen auf dem Überweisungsschein drei Zeilen mit 100 Zeichen zur Verfügung. 
Für den Spezialisten besteht eine Berichtspflicht, wenn er einen Patienten vom Hausarzt überwiesen bekommt (Einheitlicher Bewertungsmaßstab EBM 2000plus). Der Patient kann ihn davon entbinden, wenn er nicht wünscht, dass sein Hausarzt einen Befundbericht bekommt. Der Befundbericht erfolgt in der Regel schriftlich. Struktur, Inhalt und Umfang des Befundberichts sind nicht geregelt.

In einer amerikanischen Studie zur Qualität der Kommunikation zwischen Hausärzten und Spezialisten unterteilen die Autoren (Mc Phee et al. 1984) den Kommunikationsprozess in fünf Schritte:

Schritt 1: $\quad$ Arzt und Patient erkennen, dass Bedarf an einem Konsil besteht.

Schritt 2: Der behandelnde Arzt übermittelt den Grund für das Konsil und die klinische Information über den Patienten an den Spezialisten.

Schritt 3: Der hinzugezogene Arzt sieht den Patienten und erhebt einen Befund.

Schritt 4: Der Spezialist übermittelt seinen Befund und Behandlungsempfehlungen an den überweisenden Arzt.

Schritt 5: Patient, überweisender Arzt und hinzugezogener Spezialist entscheiden über das weitere Vorgehen.

Jeder dieser Schritte kann zu Schwierigkeiten führen. Der überweisende Arzt stellt den Grund für die Überweisung nicht nachvollziehbar dar oder vergisst mitzuteilen, warum er den Patienten überweist. Der hinzugezogene Arzt schafft es eventuell nicht, seine Befunde oder Empfehlungen zügig genug zu übermitteln, so dass sie nicht von Relevanz für die Therapie des Patienten sein können. Eventuell lehnt der behandelnde Arzt die Therapievorschläge des Spezialisten ab.

Schlechte Kommunikation zwischen den behandelnden Ärzten mindert die Qualität und Effektivität der Patientenversorgung. Zusätzliche Kosten entstehen, wenn Untersuchungen doppelt durchgeführt werden oder Therapien empfohlen werden, die sich bereits als unwirksam erwiesen haben. 


\section{Fragestellung und Zielsetzung}

Die folgenden Fragen sollen mit dieser Arbeit beantwortet werden:

- Wie schätzen Hausärzte selbst ihre Informationsweitergabe ein?

Die Hausärzte werden gebeten anzugeben, welche Informationen sie üblicherweise auf dem Überweisungsschein bei der Überweisung eines Patienten mit Kreuzschmerzen an den Orthopäden weitergeben.

- Welche Informationen geben Hausärzte weiter?

Die Überweisungen der Hausärzte werden auf die tatsächlichen Angaben bei der Überweisung eines Patienten mit Kreuzschmerzen an die Orthopäden untersucht.

- Welche Informationen erhalten Hausärzte von Orthopäden?

Umgekehrt werden die Angaben, die Orthopäden den Hausärzten in ihrem Befundbericht übermitteln, ausgewertet.

- Wie bewerten Hausärzte die Information, die sie erhalten?

Die Hausärzte werden nach ihrer Meinung zu den orthopädischen Befundberichten befragt. Die Bewertung erfolgt anhand eines Fragebogens.

- Aus welchen Gründen überweisen Hausärzte Patienten zum Orthopäden?

Die Hausärzte werden anhand eines Fragebogens, unabhängig von ihren Angaben auf dem Überweisungsschein, und anhand der ausgewählten Patienten nach den Gründen für die Überweisung befragt. 


\section{Material und Methoden}

\subsection{Studiendesign}

Es handelt sich um eine retrospektive, deskriptive Studie in Hausarztpraxen des Lehrpraxennetzes der Universität Göttingen. Um eine systematischen Verzerrung der Auswahl zu vermeiden, erfolgte eine Zufallsstichprobenziehung der infrage kommenden Lehrpraxen. Für ihre Mitarbeit an der Studie erhielten die Ärzte ein Honorar in Höhe von 100,-. Euro.

Es erfolgte eine schriftliche Befragung der Hausärzte, um eine Selbsteinschätzung (siehe Anlage 1) zu ihren eigenen Angaben bei der Überweisung von Kreuzschmerzpatienten zum Orthopäden zu erhalten. Zusätzlich wurden die Hausärzte gebeten, orthopädische Arztbriefe von Kreuzschmerzpatienten anhand eines Schemas (siehe Anlage 2) zu bewerten und die Patienten, die sie zum Orthopäden überwiesen haben, anhand eines Fragebogens zu charakterisieren.

\subsection{Methodische Vorüberlegungen}

Um im Hinblick auf die Studienteilnahme einer möglichen Verhaltensänderung bei der Verfassung von Überweisungstexten vorzugreifen, erfolgte die Studie retrospektiv. Das Bewusstsein, an einer Studie teilzunehmen, hätte zu besonders vorbildlich verfassten Überweisungstexten führen können.

\section{$4.3 \quad$ Pilotierung}

Im Januar 2007 fand eine Probeerhebung in einer allgemeinmedizinischen Praxis im Landkreis Göttingen zur Testung der Fragebögen statt. Die beteiligten Hausärzte gaben Anregungen zur Umstrukturierung des Fragebogens und zur Verbesserung der technischen Durchführung. In dieser Praxis erfolgte nur ein sehr geringer Anteil der Überweisungen an Orthopäden aufgrund von Kreuzschmerzen. Bei insgesamt 50 orthopädischen Überweisungen wurden lediglich 5 Patienten aufgrund von Kreuzschmerzen überwiesen. Daraufhin führten wir eine zweite Probeerhebung in einer städtischen hausärztlichen Praxis in Göttingen im Februar 2007 durch. Es wurden ausreichend viele Überweisungen aufgrund von Kreuzschmerzen gefunden. 
Bei insgesamt 60 Überweisungen an Orthopäden erfolgten 15 Überweisungen aufgrund von Kreuzschmerzen.

\subsection{Auswahlverfahren der Praxen}

Aus allen allgemeinmedizinischen Praxen des Lehrärztenetzes der Universität Göttingen, das über 120 Praxen umfasst, wurden per Zufall 62 Praxen ausgewählt (siehe Abbildung 1). Die Stichprobenziehung erfolgte mit „Procedure Normal“ (siehe Anlage 3) des Statistikprogramms SAS.

Einschlusskriterium für die Teilnahme an der Studie war die EDV-Dokumentation der Überweisung. Für die Durchführung dieser Studie waren außerdem die Dokumentationen des Überweisungstextes und des orthopädischen Arztbriefes notwendig.

\subsection{Rekrutierung der Hausärzte}

Im Februar 2007 wurden die für die Studie per Zufall ausgewählten Hausärzte angeschrieben und über das Ziel der Studie und den damit verbundenen Arbeitsaufwand in der Praxis informiert. Sie wurden gebeten, den angehängten Rückantwortbogen per Fax, E-Mail oder postalisch an die Abteilung Allgemeinmedizin zurückzusenden, wenn sie bereit waren teilzunehmen oder wenn sie Interesse hatten, aber vorab weitere Informationen wünschten. In einer zweiten Runde (Nachrekrutierung) wurden 20 weitere Ärzte angeschrieben. Es wurden die ersten und die letzten 10 Ärzte aus der alphabetischen Liste der Lehrärzte ausgewählt, sofern sie nicht schon in der ersten Rekrutierungsphase angeschrieben wurden.

\subsection{Datenerhebung in der Praxis}

Die Datenerhebung fand vom März bis Juli 2007 in Göttingen, Bremen, Kassel und Büchenbeuren statt. 
Pro Praxis wurde mit Hilfe der Praxis - EDV nach Überweisungen zum Orthopäden im Quartal IV/2006 (Oktober bis Dezember 2006) gesucht. Diese Überweisungen wurden manuell durchsucht nach Überweisungen aufgrund von Kreuzschmerzen. Da bei vielen Überweisungen lediglich „a.W.d.P.“ (auf Wunsch des Patienten) auf dem Überweisungsschein stand, wurde ebenfalls der orthopädische Arztbrief auf die Diagnosen „Lumbago“, „degeneratives LWS - Syndrom“ oder ähnliches durchsucht. Ausgewählt wurden ausschließlich die Überweisungen von Kreuzschmerzpatienten an Orthopäden. Diese sowie die dazugehörigen orthopädischen Befundbriefe wurden ausgedruckt und anonymisiert.

Jede mit Hilfe der EDV identifizierte Überweisung und der dazugehörige Arztbrief erhielten eine Nummer (Patientencode). Die Patientendaten (Name, Adresse, Krankenkasse, Geburtsdatum) auf der Überweisung und dem orthopädischen Arztbrief wurden noch in der Praxis unkenntlich gemacht. Lediglich das Alter und das Geschlecht des Patienten wurden notiert.

\subsection{Arztfragebögen}

Es erfolgte eine schriftliche Befragung der Hausärzte, um eine Selbsteinschätzung (Anlage 1) zu ihren eigenen Angaben bei der Überweisung von Kreuzschmerzpatienten zum Orthopäden zu erhalten. Zusätzlich wurden die Hausärzte gebeten, orthopädische Arztbriefe von Kreuzschmerzpatienten anhand eines Schemas (Anlage 2) zu bewerten und die Patienten, die sie zum Orthopäden überwiesen haben, anhand eines Fragebogens zu charakterisieren

Die Inhalte des selbstentwickelten Selbsteinschätzungsbogens (siehe Anlage 1) zum Überweisungsverhalten der Hausärzte orientieren sich an einer Empfehlung des Netzwerks "Integrierte Versorgung Kreuzschmerz" der IGOST (Interdisziplinäre Gesellschaft für orthopädische und unfallchirurgische Schmerztherapie) (Harter et al. 2005). Diese Empfehlung wurde für einen strukturierten Überweisungsbogen vom Hausarzt zum Orthopäden durch Orthopäden entwickelt und spiegelt daher die Wünsche der Orthopäden hinsichtlich hausärztlicher Angaben auf dem Überweisungsschein wider.

Der Fragebogen enthält Angaben $z u$ den Bereichen Schmerzdauer, Schmerzcharakter, Schmerzausstrahlung, Kraftprüfung, Beweglichkeit, bisheriger 
Therapie, Nebendiagnosen und die Frage, ob der Hausarzt dem Orthopäden einen klaren Arbeitsauftrag gibt.

Es gibt fünf Kategorien (von „trifft völlig zu“ bis "trifft nicht zu“), in die der Hausarzt sein Überweisungsverhalten einordnen kann.

Auf einem zweiten, ebenfalls selbstentwickelten Fragebogen (Anlage 2) wird die Zufriedenheit des Hausarztes mit dem orthopädischen Arztbrief hinsichtlich Struktur, Diagnose, Befunddokumentation, Angaben zu durchgeführter Diagnostik und Therapie, Angaben zu weiterer Therapie und Lesbarkeit abgefragt. Außerdem wird um eine Gesamtbeurteilung des orthopädischen Arztbriefes gebeten. Bei dem Beurteilungsbogen für den orthopädischen Befundbericht gibt es ebenfalls fünf Kategorien von „sehr zufrieden“ bis „sehr unzufrieden“ sowie die Kategorie „weiß nicht/trifft nicht zu".

Zusätzlich wird der Arzt gebeten anzugeben, ob die Initiative für die Überweisung von inm oder vom Patienten ausging. Es werden die Gründe für die Überweisung abgefragt. Als mögliche Überweisungsgründe sind vorgegeben: Wunsch nach Diagnoseüberprüfung, Chronifizierungspotential der Kreuzschmerzen, psychosoziale Belastung, Rentenwunsch des Patienten, Überweisung aus forensischen Gründen oder aus Budgetgründen. Eventuell zweifelt der Patient an der Kompetenz des Hausarztes und möchte seine Diagnose oder Therapiemöglichkeiten von einem Spezialisten bestätigt bekommen. Bei diesem Fragebogen gibt es zwei Kategorien (,trifft zu“ und "trifft nicht zu“ sowie die Antwortmöglichkeit „nicht bekannt").

\subsection{Inhaltliche Analyse der Arztbriefe}

Die Überweisungstexte der Hausärzte und die orthopädischen Arztbriefe wurden inhaltlich analysiert. Machte der Hausarzt Angaben auf dem Überweisungsschein hinsichtlich Diagnose, Nebendiagnosen, Schmerzdauer, Schmerzausstrahlung, Befund oder bisherige Therapie? Worüber berichtet der Orthopäde in seinem Arztbrief? Welche Untersuchungen wurden durchgeführt und welche Therapie empfohlen? Wird eine Wiedervorstellung beim Orthopäden gewünscht? 


\subsection{Ethik und Datenschutz}

Die Ethikkommission der Universität Göttingen hat in ihrer Sitzung im November 2006 den Antrag zur Durchführung der Studie geprüft und keine grundsätzlichen ethischen oder rechtlichen Bedenken geäußert (Anlage 5). Die Patientendaten wurden noch in der Praxis des jeweiligen Hausarztes anonymisiert. Jede Praxis, jede Überweisung sowie jeder Brief erhielten zur Zuordnung Codenummern.

\subsection{Auswertung}

Ausgewertet wurden die Daten mit dem Statistikprogramm SAS. Zur Anwendung kamen ausschließlich deskriptive Methoden. 


\section{Ergebnisse}

\subsection{Praxenrekrutierung}

In einer ersten Rekrutierungswelle wurden 62 Praxen angeschrieben. Aufgrund des geringen Rücklaufes wurden 20 weitere Praxen angeschrieben und um ihre Teilnahme an der Studie gebeten.

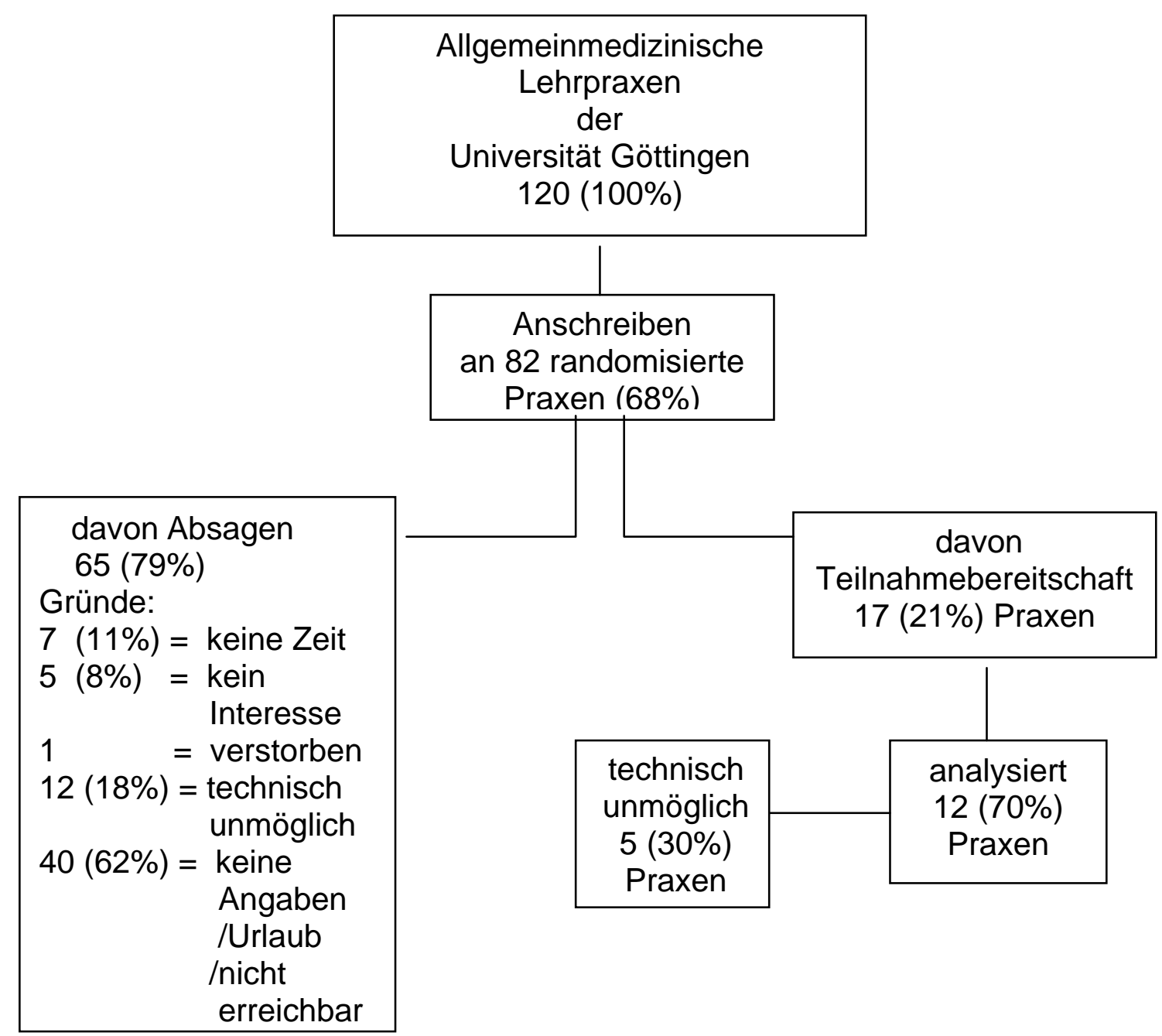

Abbildung 1: Rekrutierungsverlauf der teilnehmenden Hausarztpraxen

Es erklärten sich 17 (21\%) Praxen bereit an der Studie teilzunehmen. Aus technischen Gründen schieden 5 Praxen aus. Schließlich nahmen 12 (70\%) der teilnahmebereiten Praxen an der Studie teil. Davon stammten 7 Praxen aus der ersten und 5 Praxen aus der zweiten Rekrutierungsphase. 
Nach fünf Wochen wurden die 65 Praxen, die bisher nicht geantwortet hatten, angerufen und nach den Gründen für ihre Nicht-Teilnahme befragt. Von diesen gaben 7 Ärzte an, keine Zeit für eine Teilnahme zu haben. Für 5 Ärzte war kein Interesse an dem Thema der Grund für ihre Nicht-Teilnahme. Ein Arzt war verstorben. Aus technischen Gründen konnten 12 Ärzte nicht teilnehmen. Von 40 Ärzten konnten keine Angaben erhoben werden, da sie telefonisch nicht erreichbar oder die Praxen urlaubsbedingt geschlossen waren. Der Rekrutierungsablauf ist in Abbildung 1 dargestellt.

\subsection{Beschreibung der Praxen}

Es geben 5\% der Hausarztpraxen an, durchschnittlich >1500 Scheine/Quartal zu bearbeiten, $42 \%$ haben 1000-1500 Scheine/Quartal und 33\% 500-1000 Scheine/Quartal. Die Niederlassungsdauer der beteiligten Ärzte variiert zwischen 6 und 26 Jahren, bei einer durchschnittlichen Niederlassungsdauer von 16,8 Jahren. Häufige relevante Zusatzbezeichnungen oder Praxisschwerpunkte sind Chirotherapie $(n=4)$, Schmerztherapie $(n=2)$ oder Sportmedizin $(n=3)$.

Die 13 beteiligten Hausärzte aus 12 Praxen sind zwischen 40 und 58 Jahre alt (Durchschnittsalter 50,2 Jahre). Bis auf zwei teilnehmende Ärztinnen sind alle teilnehmenden Hausärzte männlich.

\subsection{Beschreibung der gefundenen Überweisungen/Patienten}

Von insgesamt 911 Überweisungen an die Orthopädie wurden 34\% (n=312) der Patienten aufgrund von Kreuzschmerzen überwiesen. Ausgewertet werden konnten 114 orthopädische Briefe. Diese Briefe stammen aus insgesamt zwölf Hausarztpraxen. Pro Praxis waren zwischen 4 und 24 Briefe mit Überweisungen pro Quartal vorhanden (Durchschnitt 9,5 Briefe/Praxis/Quartal).

Aufgrund von Kreuzschmerzen wurden zwischen 12 und 54 Patienten pro Praxis im Beobachtungsquartal überwiesen. Der Durchschnitt lag bei 26 Patienten/Praxis. 


\subsection{Selbsteinschätzung der Hausärzte}

Die Selbsteinschätzung der Hausärzte bei ihren Angaben auf der Überweisung zum Orthopäden ist in Abbildung 2 dargestellt. Von den befragten Hausärzten geben $50 \%$ an, dass es völlig bzw. eher zutrifft, dass sie Angaben zu Nebendiagnosen machen. 67\% der Hausärzte schätzen, dass sie Angaben zur bisherigen Therapie auf dem Überweisungsschein machen. Ebenfalls 67\% der Hausärzte schätzen, dass sie eher keine Angaben zur Beweglichkeit des Patienten auf dem Überweisungsschein machen. Ganze 75\% meinen, dass sie keine Angaben zur Kraftprüfung machen.

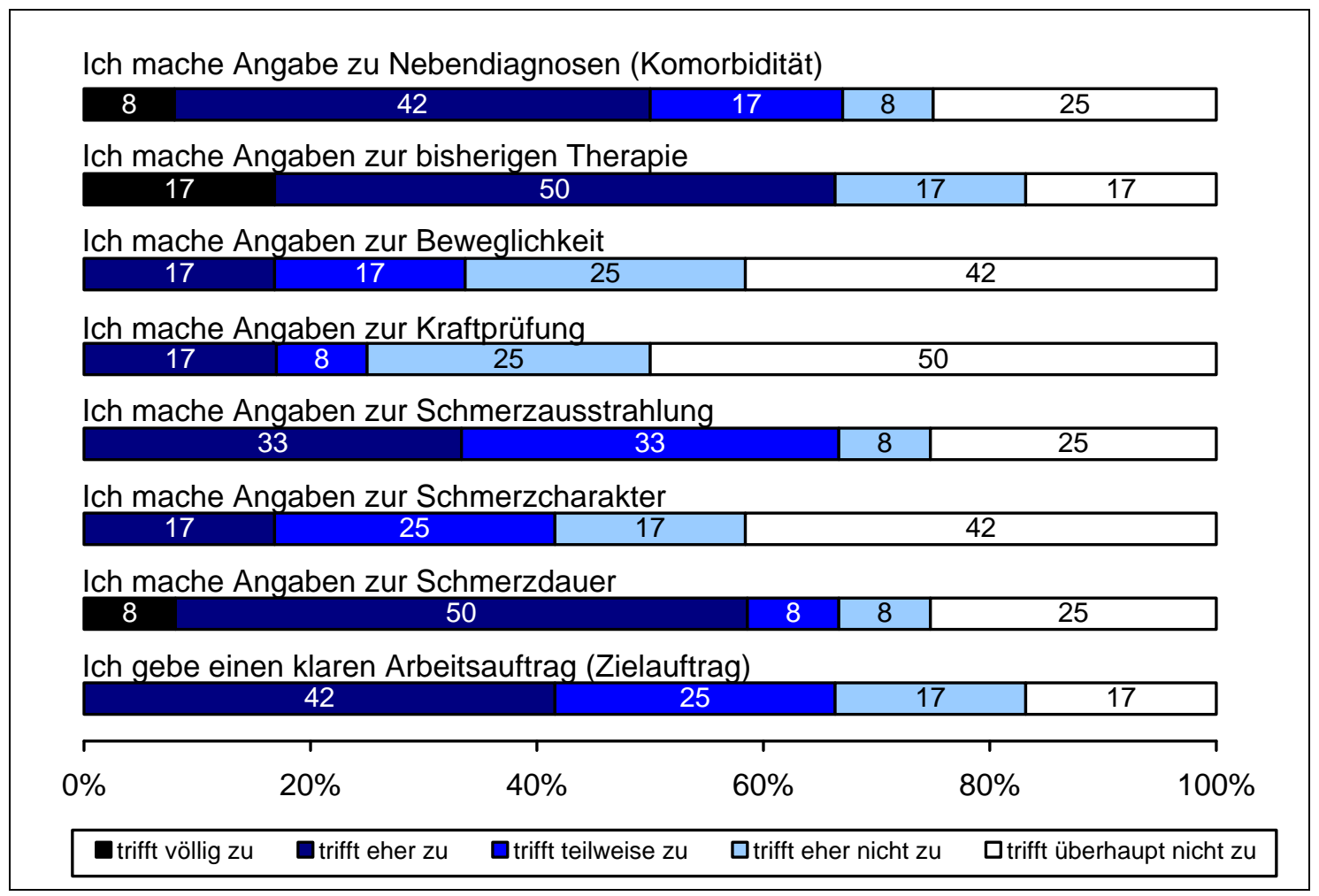

Abbildung 2: Selbsteinschätzung der Hausärzte in Bezug auf ihre Angaben auf der Überweisung (Angaben in \%)

Angaben zur Schmerzausstrahlung geben 33\% eher nicht bzw. nicht an. Ebenfalls 33\% der beteiligten Ärzte schätzen, dass sie keine bzw. eher keine Angaben zur Schmerzausstrahlung machen. Hinsichtlich des Schmerzcharakters machen 59\% nach eigener Einschätzung eher keine bzw. keine Angaben auf dem Überweisungsschein. Immerhin 58\% der Hausärzte sagen dagegen, dass sie 
Angaben zur Schmerzdauer machen. Einen klaren Arbeitsauftrag geben nach eigener Einschätzung 42\% der Hausärzte.

\subsection{Angaben der Hausärzte auf dem Überweisungsschein}

Eine Diagnose geben $82 \%$ der Hausärzte auf den Überweisungen an. Über Nebendiagnosen der Patienten berichten lediglich 30\%. Noch seltener finden sich Angaben zur Schmerzdauer (6\%), Schmerzausstrahlung (1\%) oder zum Schmerzcharakter (1\%). Einen Untersuchungsbefund geben 14\% der Hausärzte auf dem Überweisungsbogen an. Angaben zur bisherigen Therapie erfolgen sogar nur auf $5 \%$ der Überweisungen. Die Ergebnisse der Auswertung der Überweisungsscheine sind in Abbildung 3 dargestellt.

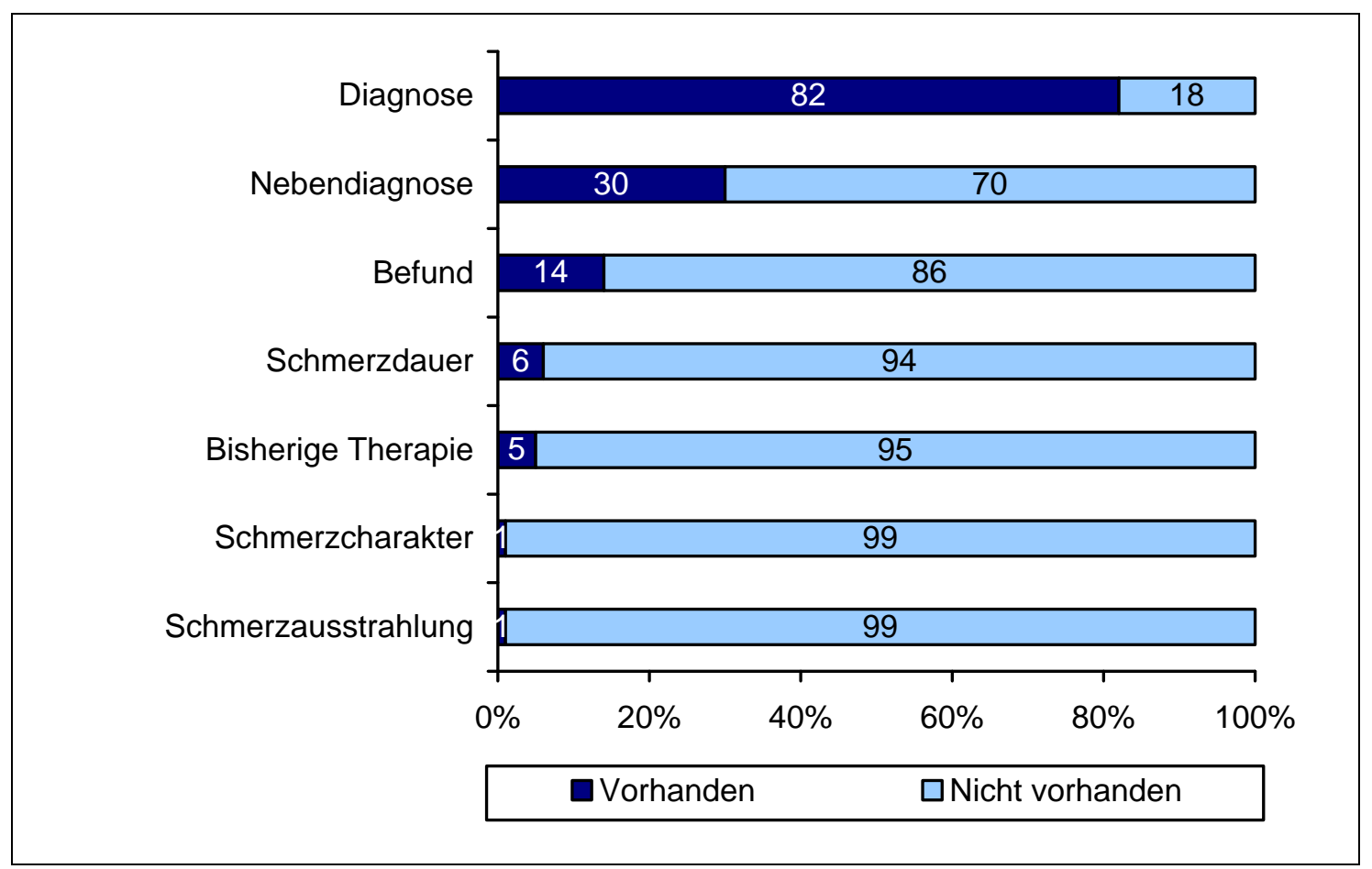

Abbildung 3: Angaben der Hausärzte auf dem Überweisungsschein (Angaben in \%) 


\subsection{Patientencharakteristika und Gründe für die Überweisung}

Die Arztbriefe beziehen sich auf 114 Patienten, die durchschnittlich 55 Jahre (von 15 Jahren bis 89 Jahren) alt sind. Der Median beträgt 53,5 und der Interquartilenrang 43-70 Jahre. Die Patienten sind überwiegend weiblich (61\%).

Vom Arzt geht die Initiative zur Überweisung zum Orthopäden lediglich zu 41\% aus. In 73\% der Fälle haben die Patienten bereits seit mehr als 4 Wochen Beschwerden.

Alle folgenden Angaben über die Patienten sind Einschätzungen ihrer behandelnden Ärzte (Abbildung 4).

Patient hat $>4$ Wochen Beschwerden

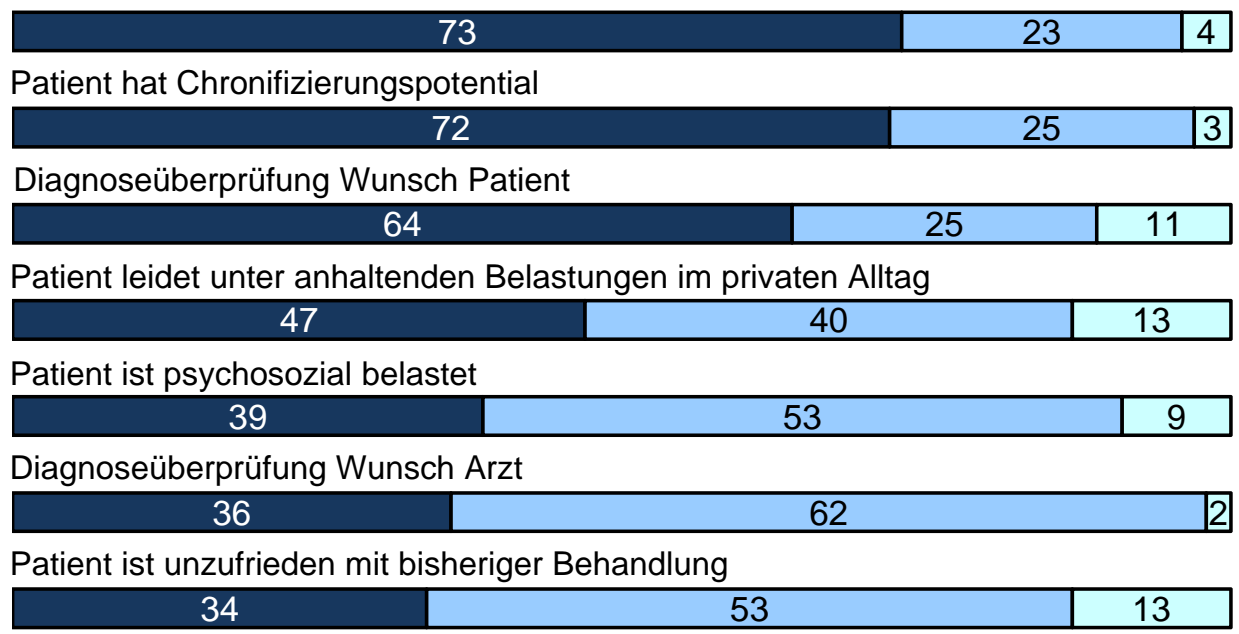

Arzt: "Ich konnte dem Patienten nicht mehr helfen."

\begin{tabular}{|c|c|c|}
\hline 29 & 70 & \\
\hline \multicolumn{3}{|c|}{ Arzt: "Ich möchte mich vom Patienten entlasten." } \\
\hline 25 & 75 & \\
\hline \multicolumn{3}{|c|}{ Patient leidet unter belastender Arbeitssituation } \\
\hline 23 & & 12 \\
\hline
\end{tabular}

Patient soll erkennen, dass die therapeutischen Möglichkeiten ausgeschöpft sind

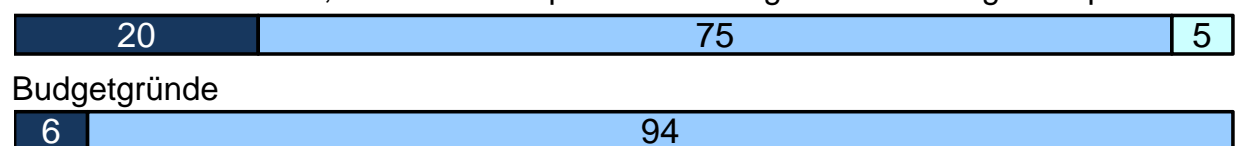

Patient hat Rentenwunsch

\begin{tabular}{|c|c|c|}
\hline 5 & 86 & 9 \\
\hline
\end{tabular}

Forensische Gründe

\begin{tabular}{|l|l|}
\hline 4 & 96 \\
\hline
\end{tabular}

$\begin{array}{lllll}0 \% & 20 \% & 40 \% & 60 \% & 80 \%\end{array}$

\section{$\square$ trifft zu $\quad \square$ trifft nicht zu $\quad \square$ weiß nicht}

Abbildung 4: Gründe für die Überweisung zum Orthopäden (Angaben in \%) 
Die Hausärzte sehen bei $72 \%$ der Patienten Chronifizierungspotential. Bei $64 \%$ der Patienten ist der Grund für die Überweisung eine Diagnoseüberprüfung auf Wunsch des Patienten. Auf Wunsch des Arztes erfolgt die Überweisung nur in 36\% der Fälle. Unter anhaltenden privaten Belastungen leiden $47 \%$ der Patienten und $39 \%$ sind laut Arzt psychosozial belastet. Rund 23\% der überwiesenen Patienten leiden nach Einschätzung ihres Hausarztes unter einer belastenden Arbeitssituation. Rentenwunsch als Grund für die Überweisung spielt nur in 5\% der Fälle eine Rolle. Unzufriedenheit mit der bisherigen Behandlung vermuten die Ärzte in 34\% der Überweisungen. Von den Hausärzten wollen sich $25 \%$ durch die Überweisung vom Patienten entlasten und 29\% überweisen, da sie meinen, dem Patienten nicht mehr helfen zu können. Von den teilnehmenden Ärzten geben $20 \%$ als Überweisungsgrund an der Patient solle erkennen, dass die diagnostischen und therapeutischen Möglichkeiten ausgeschöpft sind. Lediglich in 4\% der Fälle erfolgt die Überweisung aus forensischen Gründen.

\subsection{Auswertung der orthopädischen Arztbriefe}

Die 114 ausgewerteten, orthopädischen Befundberichte stammen von 34 unterschiedlichen Orthopäden. Die Zahl der Briefe pro Orthopäde variiert zwischen 1 und 12, im Durchschnitt beträgt sie 3 Briefe pro Orthopäde.

Die Orthopäden machen in ihrem Arztbrief in 99\% der Fälle Angaben zur Diagnose und geben in 61\% Nebendiagnosen an. Die Vorstellung der Patienten erfolgt in 65\% der Fälle ausschließlich aufgrund von Kreuzschmerzen. Über ihren körperlichen Untersuchungsbefund berichten sie in 89\% der Fälle. Bei 56\% der Befundberichte finden sich Angaben über die Durchführung des Lasègue-Tests. Angaben zur Kraftprüfung machen nur 39\% der Orthopäden. Über die Mobilität des Patienten berichten 57\%. Über eine röntgenologische Untersuchung wird in $65 \%$ der Briefe berichtet. Angaben zur Therapie machen 97\% der Orthopäden, aber lediglich $25 \%$ schreiben, ob eine Wiedervorstellung erforderlich sei. Orthopäden machen in $46 \%$ Angaben zu Schmerzdauer und Schmerzausstrahlung. Angaben zum Schmerzcharakter erfolgen dagegen nur bei $16 \%$ der Patienten. Über eine psychosoziale Anamnese wird in $10 \%$ der Fälle berichtet. Diese Angaben der Orthopäden im Befundbericht sind in Abbildung 5 dargestellt. 


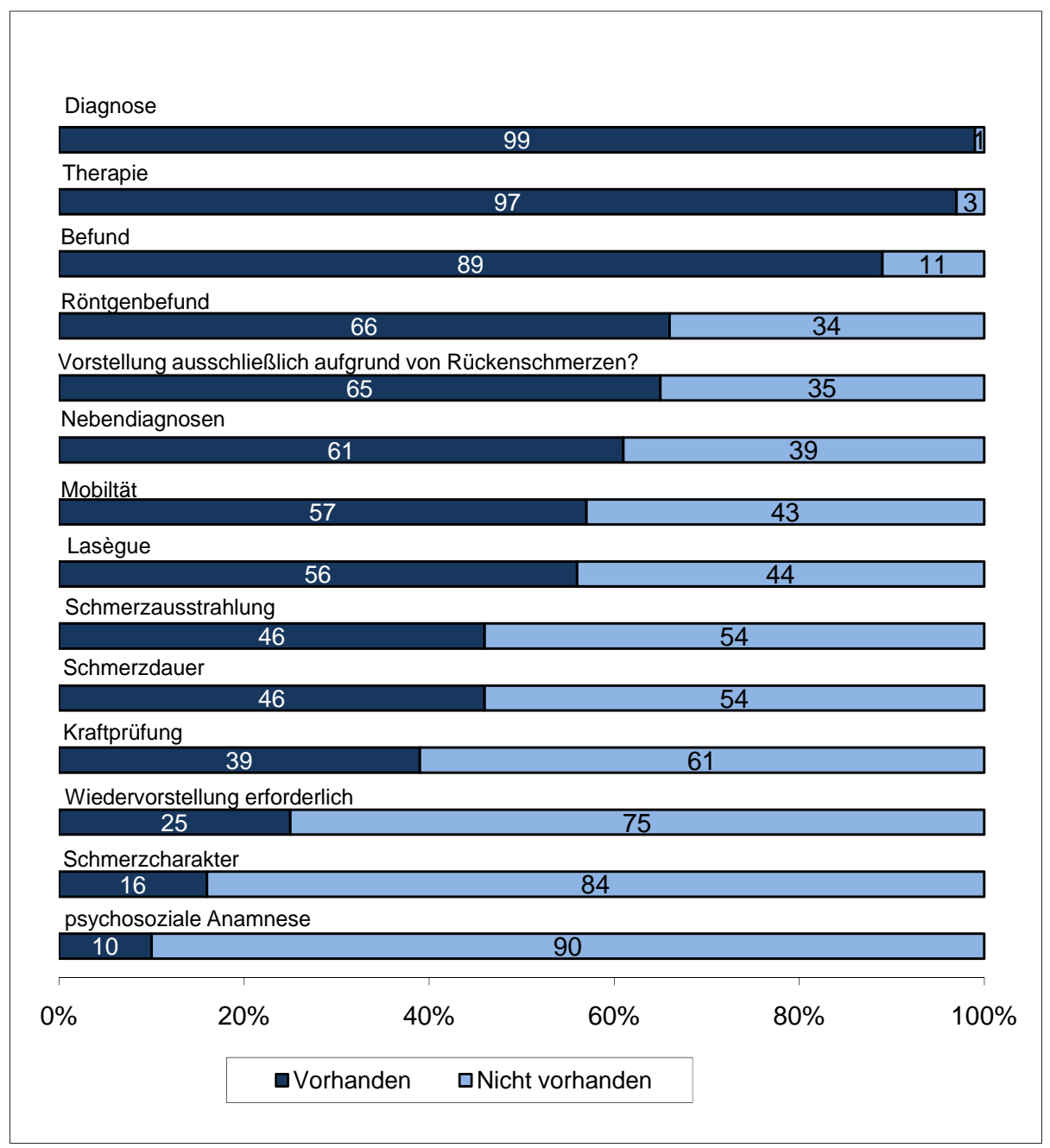

Abbildung 5: Angaben der Orthopäden im Befundbericht (Angaben in \%)

Als therapeutische Maßnahmen werden verordnet: Krankengymnastik (45\%), ein Medikament (31\%), Injektion eines Medikamentes (30\%), Chirotherapie (28\%), physikalische Therapie (25\%), Massage (9\%) oder eine Orthese (5\%).

Die Mehrheit der orthopädischen Befundberichte (85\%) ist strukturiert in Diagnose, Befund und Therapie. Das Datum des Diktates ist bei 97\% der Briefe vorhanden. Angaben zum Zeitpunkt des Praxisbesuches des Patienten erfolgen dagegen nur in 53\% der Fälle. 45\% der orthopädischen Befundberichte werden noch am Tag des Praxisbesuches diktiert. Weitere 31\% werden in den folgenden zwei Tagen diktiert. Insgesamt reicht die Zeitspanne zwischen Praxisbesuch und Diktat des Briefes von 0 (Diktat erfolgt noch am selben Tag) bis zu 46 Tagen. 


\subsection{Bewertung der orthopädischen Arztbriefe durch die Hausärzte}

Im Folgenden werden die Angaben „sehr zufrieden“ und „zufrieden“ zur besseren Lesbarkeit als „zufrieden“ zusammengefasst. „Sehr unzufrieden“ und „unzufrieden“ werden zu „unzufrieden“. Die Abbildung 6 zeigt die Zufriedenheit der Hausärzte mit dem orthopädischen Befundbericht.

Mit den Therapieempfehlungen des Orthopäden zeigt sich die Mehrheit der Hausärzte (56\%) „unzufrieden“. Nur 29\% der Hausärzte sind „Zufrieden“. Die Gesamtbeurteilung der orthopädischen Arztbriefe ist breiter gestreut. Mit dem orthopädischen Arztbrief insgesamt sind 38\% „Zufrieden“, 36\% beurteilen ihn als „ausreichend“ und 26\% zeigen sich „unzufrieden“. Mit der Lesbarkeit des orthopädischen Befundberichtes sind 83\% „Zufrieden“.

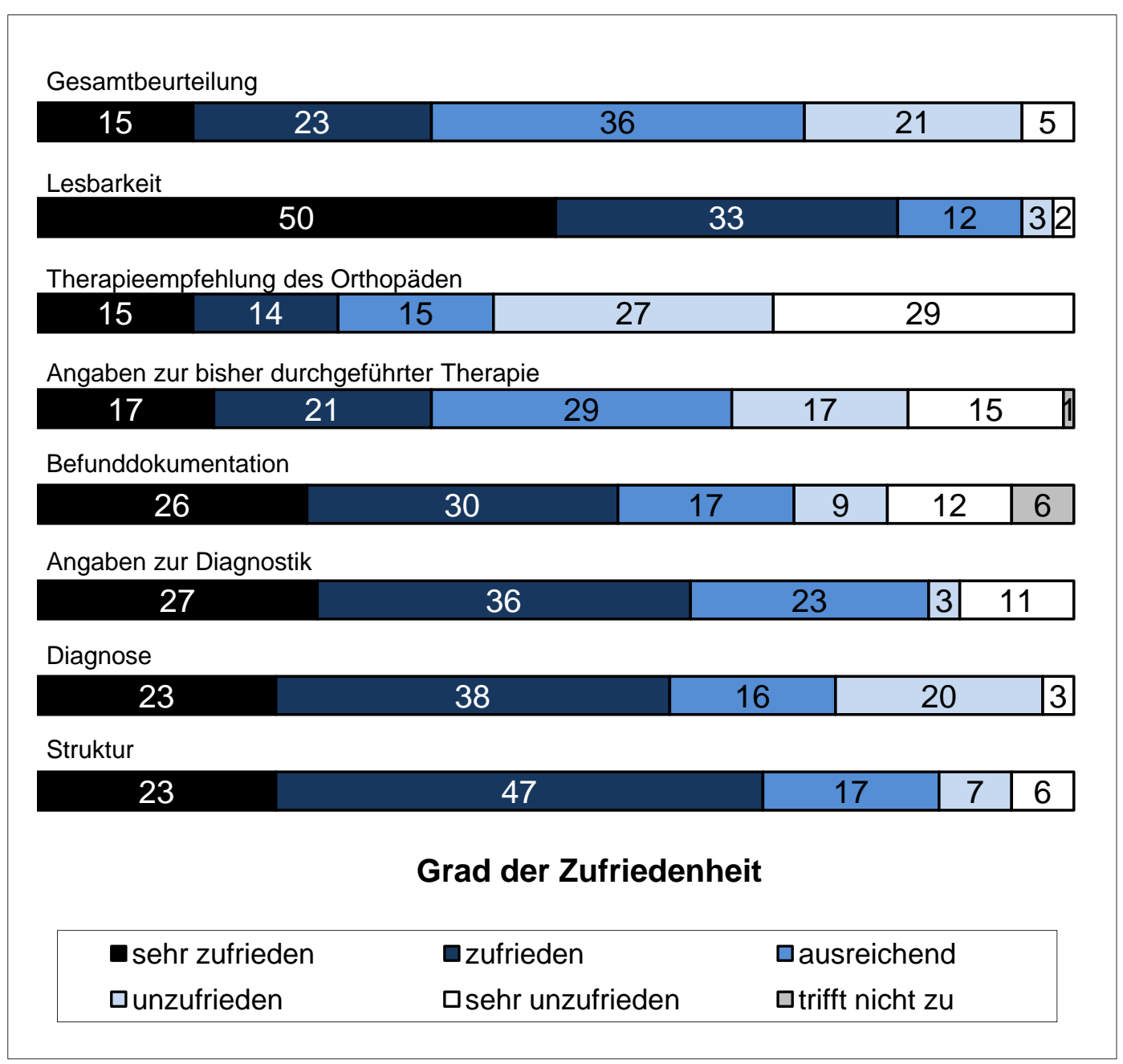

Abbildung 6: Zufriedenheit der Hausärzte mit dem orthopädischen Befundbericht (Angaben in \%) 
Mit der Befunddokumentation der Orthopäden zeigen sich 56\% der Hausärzte "zufrieden“. Lediglich 6\% gaben an „weiß nicht" (in der Abbildung 6 nicht dargestellt). Mit den Angaben der Orthopäden zur Diagnostik sind $63 \%$ der Hausärzte „zufrieden“. Ganze 61\% der Hausärzte sind mit den Angaben zur Diagnose der Orthopäden „zufrieden“. Mit der Struktur des orthopädischen Arztbriefes sind 70\% der Hausärzte „zufrieden“. Mit den Angaben zur bisher durchgeführten Therapie sind 32\% der Hausärzte „unzufrieden“, 38\% „zufrieden“ und 29\% beurteilen sie als „ausreichend“. Nicht beurteilen („weiß nicht“) können 1\% der Hausärzte die Angaben zur bisher durchgeführten Therapie. 


\section{Diskussion}

\subsection{Zusammenfassung der wichtigsten Ergebnisse}

Diese Beobachtungsstudie zeigt, dass Hausärzte nur selten über eine Diagnose hinaus Angaben auf dem Überweisungsschein machen. Ihre Selbstwahrnehmung weicht davon zum Teil erheblich ab. Der Wunsch nach einer Überweisung zum Orthopäden geht in der Mehrzahl der Fälle vom Patienten aus. Nur für ein Drittel der überwiesenen Patienten erhalten Hausärzte trotz Berichtpflicht einen Befundbericht. Mit dem orthopädischen Befundbericht sind sie insgesamt zufrieden. Unzufrieden sind sie jedoch in über der Hälfte der Fälle mit den Therapieempfehlungen. Psychosoziale Angaben fanden sich lediglich in $10 \%$ der orthopädischen Befundberichte. Die Angaben in den Befundberichten sind heterogen und entsprechen nicht immer den Empfehlungen der Interdisziplinären Gesellschaft für Orthopädische Schmerztherapie.

\subsection{Stärken und Schwächen dieser Doktorarbeit}

Diese Studie befasst sich erstmals in Deutschland speziell mit der Überweisung von Kreuzschmerzpatienten vom Hausarzt zum Orthopäden und dem damit verbundenen Befundaustausch in der ambulanten Versorgung.

Es handelt sich um eine kleine Stichprobe von Hausärzten und Orthopäden. Dennoch besteht kein Grund zur Annahme, dass die beobachtete Überweisungspraxis und Befundmitteilung stark von der üblichen Praxis in Deutschland abweicht. An der Studie nahmen engagierte Lehrärzte teil, bei denen ein überdurchschnittliches Niveau der Patientenversorgung vermutet werden könnte. Die Repräsentativität von Ärzten, die einem Lehr- und Forschungsnetzwerk angehören, ist vergleichbar mit Studienteilnehmern, die keinem Netzwerk angehören (Wetzel et al. 2005). Auch eine englische Studie, welche die Versorgung in Forschungspraxen und Nicht-Forschungspraxen verglich, konnte keine wesentlichen Unterschiede zeigen (Hammersley et al. 2002).

Die geringe Teilnehmerrate hängt mit dem Einschlusskriterium EDV-Dokumentation und der Möglichkeit der Praxen zusammen, Überweisungen zum Orthopäden mit der Software herauszufiltern. Hinzu kommt ein hoher Aufwand in der Praxis von 
mindestens 3 Stunden, um die Briefe zu identifizieren und zu kopieren, den viele Ärzte scheuten.

Einige Praxen konnten trotz EDV-Dokumentation aus technischen Gründen nicht teilnehmen. Obwohl mittlerweile aus abrechnungstechnischen Gründen mindestens 93\% der Praxen in Deutschland über einen Computer verfügen, wird meist parallel eine Papierdokumentation durchgeführt (Heidenreich et al. 2005, Gallup-Umfrage im Auftrag der Europäischen Union 2002). Auch wenn die EDV nach Überweisungen suchen kann, war es in diesen Praxen nicht immer möglich, überwiesene Patienten zu identifizieren. Der Aufwand dafür, Patienten mit einer Überweisung zum Orthopäden bei Kreuzschmerzen anhand der Befundberichte aus der Papierkartei zu identifizieren, wäre unverhältnismäßig groß bzw. unmöglich gewesen. Zusätzlich hätte es Schwierigkeiten bei der Dokumentation der Überweisungstexte gegeben, da diese handschriftlich ausgefüllt werden und daher nicht mehr nachvollzogen werden konnten.

\subsection{Kritische Betrachtung der Ergebnisse}

\subsubsection{Angaben der Hausärzte}

Im Gegensatz zu ihrer Selbstwahrnehmung machen Hausärzte selten über eine Diagnose hinaus Angaben auf dem Überweisungsschein. Hierfür gibt es mehrere mögliche Ursachen. Es gibt bisher keine anerkannten Vereinbarungen, welche Informationen genau übermittelt werden sollen. Vorschläge für standardisierte Überweisungsformulare existieren von der AOK Baden-Württemberg in Kooperation mit dem Deutschen Hausärzteverband im Rahmen des Netzwerkes für „Integrierte Versorgung von Kreuzschmerzen“ als Formular „Patientenbegleitdokumentation“ (siehe Anlage 6) für die Überweisung bei Kreuzschmerzen. Dieses Formular enthält Fragen zum Beginn der Kreuzschmerzen, zum Verlauf, zu bisherigen therapeutischen Maßnahmen und zur Fragestellung des Hausarztes, die mit der Überweisung beantwortet werden soll. Von der IGOST (Interdisziplinäre Gesellschaft für Orthopädische Schmerztherapie) gibt es ebenfalls einen Vorschlag für einen strukturierten Überweisungsbogen bei Kreuzschmerzen (Harter et al. 2005). Aus Sicht der Orthopäden sind dabei folgende Punkte wichtig: Schmerz (Dauer, Auslöser, Charakter, Ausstrahlung, Stärke), Befund, Diagnosen, veranlasste Maßnahmen, „red“, „orange“ und „yellow flags“. 
Dem gegenüber steht, dass 89\% der Hausärzte und 83\% der Spezialisten (Newton et al. 1992) es bevorzugen, einen freien Text zu schreiben anstatt ein standardisiertes Formular auszufüllen.

Auf dem üblichen Überweisungsschein (Muster 6) stehen einerseits lediglich wenige Zeilen für Informationsangaben zur Verfügung (Bundesmantelvertrag http://www.kbv.de/rechtsquellen/134.html). Ausführlichere Angaben erfordern das aufwendige Erstellen eines Überweisungsbriefes.

Häufig ging die Initiative für die Überweisung zudem nicht vom Hausarzt aus. Es fehlte folglich eine konkrete Fragestellung und es kann vermutet werden, dass die Motivation detaillierte Angaben zu machen, deshalb geringer war.

In Deutschland gibt es formal kein Primärarztsystem zur Patientensteuerung. Die Einführung der Praxisgebühr im Januar 2004 hat dazu geführt, dass Patienten sich vermehrt Überweisungen bei ihrem Hausarzt abholen (Merten 2008). Die Anzahl der Überweisungen beim Hausarzt ist von 2003 zu 2004 in Niedersachsen um das Doppelte angestiegen. Allerdings sind die Rahmenbedingungen so, dass der Hausarzt keinen finanziellen Anreiz für ein wirkliches "gatekeeping“ hat (Böcken 2006). Es fehlen strukturelle Voraussetzungen, wie z.B. ein Listensystem mit fest eingeschriebenen Patienten für einen Hausarzt. Überweisungswünsche werden daher zum Teil ohne direkten Arzt-Patienten-Kontakt erfüllt.

\subsubsection{Gründe für die Überweisung}

Der Wunsch nach einer Überweisung zum Orthopäden ging nach Meinung der Hausärzte in der Mehrzahl der Fälle vom Patienten aus. Die Mehrheit der Patienten (73\%) litt unter chronischen Kreuzschmerzen. Die DEGAM-Leitlinien zur Behandlung von Kreuzschmerzen (DEGAM 2003) empfehlen bei Patienten mit mehr als vier Wochen persistierenden Schmerzen ohne ausreichende Besserung eine fachärztliche Befundkontrolle. Bei den meisten Patienten in dieser Studie liegt wahrscheinlich nicht die erste Episode von Kreuzschmerzen vor. Daher ist anzunehmen, dass ein großer Teil bereits früher fachärztlich untersucht wurde. Der Begriff „rezidivierende Kreuzschmerzen“ ist in der internationalen Literatur nicht eindeutig definiert. Es ist unklar, wie oft und in welchen Abständen Patienten mit rezidivierenden Kreuzschmerzen sowohl primärärztlich als auch fachärztlich reevaluiert werden sollen. 
Bei einem Drittel der Patienten vermuten die Hausärzte Unzufriedenheit mit der bisherigen Behandlung. Sie nehmen an, dass sich diese Patienten durch die Überweisung eine Diagnoseüberprüfung und neue Therapiemöglichkeiten erhoffen. Die Hausärzte fühlen sich in einem Drittel der Fälle nicht mehr in der Lage, dem Patienten zu helfen. Ein Drittel der Hausärzte wünscht sich ebenfalls eine Diagnoseüberprüfung durch den Orthopäden. Aus forensischen Gründen überweisen lediglich 4\%. Dies zeigt, dass die diagnostische Unsicherheit bei Kreuzschmerzen relativ gering ist. Fast drei Viertel der überwiesenen Patienten leiden unter chronischen Kreuzschmerzen. Chronische Rückenschmerzpatienten sind in der ambulanten Regelversorgung kaum optimal zu versorgen (Lang et al. 2002). Zur Entlastung oder aus Budgetgründen überwiesen zu haben, gaben nur wenige Hausärzte an. Hier könnte ein Erwünschtheit-Bias vorliegen.

\subsubsection{Fehlende Befundberichte}

Lediglich für ein Drittel der überwiesenen Patienten erhielten die Hausärzte trotz Berichtpflicht einen Befundbericht (EBM 2000plus). Gründe dafür könnten sein, dass Patienten der Befundweitergabe an den Hausarzt widersprochen haben. Es kann nicht ausgeschlossen werden, dass überwiesene Patienten den Termin beim Orthopäden nicht wahrgenommen haben, da sich die Beschwerden in der Zwischenzeit gebessert haben. Sollten die Briefe sehr verzögert, d.h. später als drei Monate nach dem Besuch des Patienten beim Orthopäden eingetroffen sein, waren sie zum Zeitpunkt der Datenerhebung noch nicht in der Praxis angekommen. Die Briefe könnten auch aus anderen Gründen in der Praxis nicht auffindbar gewesen sein. Der Orthopäde könnte die Behandlung des Patienten selbstständig weitergeführt oder zu einem weiteren Spezialisten überwiesen haben. Diese Begründungen könnten mögliche Ursachen sein, können aber den sehr geringen Anteil an auffindbaren Befundberichten nicht ausreichend erklären. Dies schien die teilnehmenden Hausärzte nicht zu stören, da keiner den geringen Rücklauf von Befundberichten bisher bemerkt hatte.

\subsubsection{Inhalt und Qualität der orthopädischen Briefe}

Um den individuellen Arztbrief nach EBM 01601 abrechnen zu können, muss er folgende Informationen enthalten: Anamnese, Befund(e), epikritische Bewertung und eine Therapieempfehlung (Bundesmantelvertrag). 
Studien zur Chronifizierung haben gezeigt, dass psychosoziale Faktoren für den Krankheitsverlauf von Kreuzschmerzen eine entscheidende Rolle spielen (Airaksinen et al. 2006). Viele der Patienten mit Kreuzschmerzen sind psychosozial belastet oder leiden unter anhaltenden Belastungen im privaten Alltag. Trotz der im Vergleich zu somatischen Befunden höheren Bedeutung psychosozialer Befunde für die Chronifizierung (Pfingsten und Schöps 2004) wird in den orthopädischen Briefen lediglich in $10 \%$ der Fälle auf sie eingegangen. Für die Behandlung von Rückenschmerzpatienten ist eine Berücksichtigung dieser Faktoren auch durch somatisch ausgerichtete Orthopäden wichtig.

Mit dem orthopädischen Befundbericht waren die Hausärzte insgesamt zufrieden. In mehr als der Hälfte der Fälle zeigen sie sich jedoch unzufrieden mit den Behandlungsempfehlungen der Orthopäden. Diese Unzufriedenheit könnte die Begrenztheit der Therapiemöglichkeiten bei Patienten mit chronischen Kreuzschmerzen widerspiegeln. Dieses könnte auch erklären, warum die geringe Befundberichtsquote die Hausärzte nicht zu stören schien. 97\%, der von uns untersuchten orthopädischen Befundberichte, enthalten Angaben zur weiteren Therapie. Im Regelfall verordnen Orthopäden auch die von Ihnen empfohlenen Medikamente bzw. Physiotherapie. Budgetgründe waren nur für 6\% der Hausärzte ein Grund für eine Überweisung. Die Leitlinienkongruenz der Therapieempfehlungen kann nur eingeschränkt beurteilt werden, da nicht genügend Patientendaten vorliegen. Ein hoher Anteil von Patienten erhielt jedoch passive Maßnahmen, die von den europäischen Leitlinien nicht empfohlen werden (Becker et al. 2004, Airaksinen et al. 2006).

\subsection{Vergleich mit der internationalen Literatur}

Nach meiner Literaturrecherche ist dieses die erste Studie in Deutschland, die sich speziell mit der Überweisung von Kreuzschmerzpatienten vom Hausarzt zum Orthopäden und dem damit verbundenen Befundaustausch in der ambulanten Versorgung befasst.

In Großbritannien wurde eine ähnliche Studie zur Kommunikation zwischen Orthopäden und Hausärzten durchgeführt (Jacobs und Pringle 1990). Die Patienten wurden von niedergelassenen Hausärzten an Orthopäden in Lehrkrankenhäusern (Outpatient Clinic) überwiesen. $14 \%$ der Patienten wurden aufgrund von 
Kreuzschmerzen überwiesen. Die Autoren stellen fest, dass in den orthopädischen Befundberichten häufig nicht auf die Überweisungstexte eingegangen wird. Mehr als die Hälfte der in der Überweisung gestellten Fragen bleiben unbeantwortet. Sowohl der Überweisungsbrief des Hausarztes als auch die Antworten der Orthopäden sind inhaltlich nicht zufriedenstellend. Im Vergleich machen britische Hausärzte deutlich mehr Angaben auf ihrer Überweisung als deutsche Hausärzte. Beispielsweise geben 94,1\% der britischen Hausärzte einen Überweisungsgrund an. Dagegen geben nur 82\% der deutschen Ärzte eine Diagnose an. Angaben zur bisherigen Therapie machen in Großbritannien 33\% und in Deutschland lediglich 5\%. Einen Untersuchungsbefund geben in Großbritannien 59,7\% der Hausärzte und in Deutschland nur 14\% an. Die Selbsteinschätzung der Hausärzte in Deutschland hinsichtlich ihrer Angaben auf der Überweisung liegt deutlich über den tatsächlich gefundenen Angaben. Beim direkten Vergleich muss jedoch berücksichtigt werden, dass es sich um Überweisungsbriefe zwischen Hausärzten und einer orthopädischen Klinik handelt und in Großbritannien ein individuell verfasster Überweisungsbrief üblich ist.

In einer weiteren britischen Studie, die sich mit der Kommunikation zwischen Hausärzten und Orthopäden befasst, werden unterschiedliche Bedürfnisse dieser beiden Gruppen an den Inhalt der Arztbriefes festgestellt. Am Wichtigsten sind für Hausärzte Diagnose, bisherige Behandlung und ein Behandlungsplan. Orthopäden legen ihre Schwerpunkte auf Schmerzdauer und Lokalisation. Als Optimum wurde ein Antwortbrief angesehen, der alle oben genannten Punkte enthält. Lediglich 26\% der untersuchten orthopädischen Briefe enthielten diese Informationen jedoch vollständig (Hook et al. 2006). Die in dieser Studie befragten Hausärzte und Orthopäden bevorzugten eindeutig strukturierte Arztbriefe. In der eigenen Studie machen Orthopäden fast immer Angaben zur Diagnose und Therapie, es zeigten sich aber Mängel bei der Anamnese und Mitteilung des Untersuchungsbefundes. Das in Deutschland benutzte Überweisungsformular (Muster 6) ist für eine strukturierte Angabe wegen des geringen Platz und der mangelnden Vorstrukturierung nicht geeignet. Aus hausärztlicher Sicht ist ein krankheitsspezifisches Überweisungsformular nicht wünschenswert, da Kreuzschmerzen nur einen kleinen Teil des Versorgungsspektrums ausmachen (Wey 2004). 
In einer niederländischen Studie versorgen umgekehrt lediglich 35\% der Hausärzte den Spezialist in ihrem Überweisungsbrief mit klinischer Information. Die Studie zeigte deutliche Defizite der Informationsübermittlung auf beiden Seiten. Sowohl die Befundberichte der Spezialisten als auch die hausärztlichen Überweisungsbriefe seien verbesserungsfähig. Untersucht wurden 637 Überweisungsbriefe und die dazugehörigen Antwortbriefe der Spezialisten. Die Studie war jedoch nicht speziell auf die Kommunikation zwischen Orthopäden und Hausärzten ausgerichtet. Insbesondere wurde kritisiert, dass im Befundbericht nicht auf den Überweisungsbrief eingegangen wurde. So erhielten nur 13\% der Hausärzte eine explizite Antwort auf ihre Fragestellung, 62\% der Befundberichte enthielt eine implizite Antwort und in einem Viertel der Fälle erhielt der Hausarzt keine Antwort auf seine Frage (Grol et al. 2003).

Eine amerikanischen Studie zeigt, dass die Spezialisten häufiger persönlich oder telefonisch auf die Überweisung reagieren, wenn sie ebenfalls persönlich vom Hausarzt kontaktiert werden. Zudem schätzen die Hausärzte die Rücklaufquote mit $55 \%$ niedriger ein als tatsächlich Arztbriefe in der Patientenakte gefunden wurden (86\%) (McPhee et al. 1984).

In unserer Studie wurde nicht auf einen etwaigen telefonischen Kontakt eingegangen. Es kann jedoch davon ausgegangen werden, dass diese Form der Kommunikation beispielsweise aus Zeit- und Organisationsgründen eher die Ausnahme darstellt.

\subsection{Schlussfolgerungen und Ausblick}

Schlechte Kommunikation zwischen den behandelnden Ärzten mindert die Qualität und Effektivität der Patientenversorgung. Die mangelhaften Angaben auf den Überweisungsscheinen von Hausärzten sowie die hohe Zahl fehlender Befundberichte von Orthopäden signalisieren nicht so sehr eine eigentliche Kommunikationsstörung zwischen Hausärzten und Orthopäden, sondern vielmehr scheinen die bisherigen Versorgungsstrukturen und Instrumente wie der Überweisungsschein eine effektive Kommunikation zu verhindern.

Die mangelnde Kommunikation zwischen Hausärzten und Orthopäden bestätigt auf interkollegialer Ebene die vom Sachverständigenrat bereits festgestellte Unter-, Fehlund Überversorgung bei Kreuzschmerzen. Für eine strukturierte Versorgung werden 
Schnittstellendefinitionen für Überweisungen und eine klare Verteilung der Aufgaben und Verantwortlichkeiten benötigt. Bisherige nationale Leitlinien zur Versorgung von Kreuzschmerzpatienten sind meist monodisziplinär erstellt worden. Für die Implementierung von Leitlinien gilt eine multidisziplinäre Erstellung als günstiger (Breen et al. 2006). Auf lokaler Ebene gibt es Beispiele für gelungene Kooperationsmodelle zwischen Hausärzten und Orthopäden (Bonnemann et al. 2007). Im Rahmen des Programms für Nationale Versorgungsleitlinien sollen zurzeit im Auftrag der Bundesärztekammer und der kassenärztlichen Bundesvereinigung eindeutige Schnittstellen- und Kommunikationsstandards definiert werden (Ärztliches Zentrum für Qualität in der Medizin). Wie sich Kommunikation und Befundsaustausch intensivieren ließe, könnte ein zukünftiges Thema sein. Dafür wäre eine genaue Erfassung der Informationsbedürfnisse beim Befundaustausch zwischen Hausärzten und Orthopäden als Grundlage für Empfehlungen sinnvoll. Weniger sinnvoll dagegen dürfte ein krankheitsspezifisches Überweisungsformular sein, da Kreuzschmerzen in der Hausarztpraxis zwar relativ häufig sind, aber absolut nur einen kleinen Teil des Versorgungsspektrums ausmachen. Eine allgemeine Optimierung des Überweisungsformulars wäre der bessere Weg. 


\section{Zusammenfassung}

Hintergrund. Rückenschmerzen sind ein häufiger Konsultationsanlass in der hausärztlichen Praxis. Die Behandlung der Patienten erfolgt oft in Kooperation mit Orthopäden und erfordert daher einen effektiven Informationsaustausch. Ziel dieser Arbeit war es, die Kommunikation zwischen Hausärzten und Orthopäden zu untersuchen.

Material und Methoden. Hausärztliche Überweisungen und die dazu gehörenden orthopädischen Befundberichte wurden retrospektiv ausgewertet. Hausärzte nannten die Gründe für die Überweisung und bewerteten die Qualität der Befundberichte.

Ergebnisse. Insgesamt nahmen 12 von 82 Allgemeinärzten des Lehrpraxisnetzes der Universitätsmedizin Göttingen an der Studie teil. Von 911 hausärztlichen Überweisungen an die Orthopädie wurden in 3 Monaten 312 (34 \%) Patienten wegen Rückenschmerzen überwiesen. Hausärzte machten nur selten über eine Diagnose hinaus Angaben auf dem Überweisungsschein. Ihre Selbstwahrnehmung wich davon erheblich ab. Der Wunsch nach einer Überweisung zum Orthopäden ging in der Mehrzahl der Fälle (64 \%) vom Patienten aus und bei $72 \%$ wurde Chronifizierungspotential vermutet. Nur für ein Drittel der überwiesenen Patienten (114/312) erhielten Hausärzte trotz Berichtspflicht einen Bericht, der relativ wenige Befundangaben enthielt. Zumeist waren die Hausärzte damit zufrieden, aber bei 59\% der Überweisungen waren sie unzufrieden mit den Therapieempfehlungen. Psychosoziale Angaben fanden sich nur in $10 \%$ der orthopädischen Befundberichte. Die Angaben in den Befundberichten waren heterogen und entsprachen nur teilweise den Empfehlungen der Interdisziplinären Gesellschaft für Orthopädische Schmerztherapie.

Schlussfolgerung. Die mangelhaften Angaben auf den hausärztlichen Überweisungsscheinen sowie die hohe Zahl fehlender Befundberichte signalisieren nicht so sehr eine eigentliche Kommunikationsstörung zwischen Hausärzten und Orthopäden, sondern vielmehr scheinen die bisherigen Versorgungsstrukturen und Instrumente wie der Überweisungsschein eine effektive Kommunikation zu verhindern. So erklärt sich die relativ große Zufriedenheit von Hausärzten mit den inhaltlich wenig aussagekräftigen Befundberichten. Ihre Unzufriedenheit mit den 
orthopädischen Behandlungsempfehlungen reflektiert eher die beschränkten Behandlungsoptionen bei chronischen Rückenschmerzen in der ambulanten Versorgung. 


\section{Literaturverzeichnis}

Airaksinen O, Brox Jl, Cedraschi C, Hildebrandt J, Klaber-Moffett J, Kovacs F, Mannion AF, Reis S, Staal JB, Ursin H (2006): Guidelines for Chronic Low Back Pain. Chapter 4. European guidelines for the management of chronic non-specific low back pain. Eur Spine J 15, 192-300

Ärztliches Zentrum für Qualität in der Medizin: Programm für Nationale Versorgungsleitlinien http://www.leitlinien.de/leitlinienthemen/LLThema/00000179/viewitem (letzter Zugriff am 02.08.2008)

Becker A, Hildebrandt J, Müller G (2004): Europäische Leitlinien für den Umgang mit unspezifischen Kreuzschmerzen. http://schmerzambulanz.humanmedizingoettingen.de/rs_leitlinien.pdf (letzter Zugriff am 07.01.2009)

Berichtspflicht Fachärzte EBM 2000plus

http://www.kvhessen.de/Mitglieder/Abrechnung+und+Honorar/EBM/Berichtspflicht/Be sondere+Berichtspflicht+für+Ärzte-char-A.pdf (letzter Zugriff am 16.07.2008)

Bertelsmann-Stiftung (2007): Qualitätsmanagement im Versorgungsprozess von Rückenpatienten. www.bertelsmann-stiftung.de/cps/rde/xchg/SID-OA000FOA14A2C28F/bst/hs.xsl/prj_8502.htm (letzter Zugriff am 06.01.2008)

Böcken J, Braun B, Amhof R, Schnee M: Hausarztmodelle in Deutschland: Teilnehmerstruktur, Beitrittsgründe und die Koordination zum Facharzt. Gesundheitsmonitor 2006, Bertelsmann Stiftung, Gütersloh 2006

Bolten W, Kempel-Waibel A, Pforringer W (1998): Analyse der Krankheitskosten bei Kreuzschmerzen. Med Klin 93, 388-93

Bonnemann C, Bonnemann D, Hoffmann D (2007): Bielefelder Rückenmodell: Stärkere Berücksichtigung personaler und sozialer Faktoren. Dtsch Arztebl 104, 2248-51

Breen AC, van Tulder MW, Koes BW, Jensen I, Reardon R, Bronfort G (2006): Mono-disciplinary or multidisciplinary back pain guidelines? How can we achieve a common message in primary care? Eur Spine J 15, 641-7

Bundesmantelvertrag http://www.kbv.de/rechtsquellen/134.html (letzter Zugriff am 02.08.2008)

Chenot JF, Scherer M, Becker A (2003): Eine neue DEGAM-Leitlinie: Kreuzschmerzen. Z Allgemeinmed 80, 351-3

Chenot JF, Scherer M, Becker A (2006): Die körperliche Untersuchung bei Schmerzen im Lendenwirbelbereich. Z Allgemeinmed 82, 132-40

Chenot JF, Becker A, Niebling W, Kochen MM (2007): Aktualisierung der DEGAMLeitlinie Kreuzschmerzen. Z Allgemeinmed 83, 487-94 
Chenot JF, Leonhardt C, Keller S, Scherer M, Donner-Banzhoff N, Baum E, Pfingsten M, Basler HD, Kochen MM, Becker A (2008): The impact of specialist care on health service utilization in primary care patients with low back pain: a prospective cohort study. Eur J Pain 12, 275-83

DEGAM (Deutsche Gesellschaft für Allgemeinmedizin und Familienmedizin Hrsg.): Leitlinie Kreuzschmerzen. Omikronpublishing, Düsseldorf 2003

http://www.degam.de/leitlinien/3_kreuzschmerzen.html (letzter Zugriff am 16.5.2006)

Deyo RA, Weinstein DO (2001): Low back pain. N Engl J Med 344, 363-70

EBM 2000plus

http://www.kbv.de/8156.html (letzter Zugriff am 16.07.2008)

Gallup-Umfrage: Management information system Médecines généralistes. Flash Eurobarometre 126. (2002) http://ec.europa.eu/public_opinion/flash/fl126_fr.pdf (letzter Zugriff am 10.06.2008)

Gesundheitsberichterstattung 2006:

http://www.gbebund.de/gbe10/owards.prc_show_pdf?p_id=9965\&p_sprache=D\&p_uid=gast\&p_aid =21863334\&p_Ifd_nr=1 (letzter Zügriff am 30.07.2008)

Göbel H (2001): Epidemiologie und Kosten chronischer Schmerzen. Spezifische und unspezifische Kreuzschmerzen. Schmerz 15, 92-8

Grol R, Rooijackers-Lemmers N, van Kaathoven L, Wollersheim H, Mokkink H (2003): Communication at the interface: do better referral letters produce better consultant replies? Br J Gen Pract 53, 217-219

Hammersley V, Hippisley-Cox J, Wilson A, Pringle M (2002): A comparison of research general practices and their patients with other practices--a cross-sectional survey in Trent. Br J Gen Pract 52, 463-8

Harter W, Schiffdecker-Hoch F, Brunner HE, Denner A: Menschen in BewegungMultidimensionale Neuausrichtung in der Behandlung chronischer Rückenschmerzen. Deutscher Ärzteverlag, Köln 2005

Hausärzteverband Baden- Württemberg: Patientenbegleitdokument. http://www.hausaerzteverband.de/cms/uploads/media/2007_10_23_patientenbegleit doku.pdf (letzter Zugriff am 07.08.2008)

Heidenreich R, Himmel W, Böckmann H, Hummers-Pradier E, Kochen MM, Niebling W, Rogausch A, Sigle J, Wetzel D, Scheidt-Nave C (2005): Documentation of electronic patient records (EPRS) in German general practices: a telephone survey. Z Arztl Fortbild Qualitatssich 99(9), 573-80

Hensler S, Jork K (2002): Schnittstellen im Deutschen Gesundheitssystem Bedeutung für die hausärztliche Versorgung. Z Allgemeinmed 78, 219-24

Hook S E, Banister G C, Topliss C, Webb J (2006): Letters and notes in orthopaedic surgery. Ann R Coll Surg Engl 88, 292-296 
Jacobs LGH, Pringle MA (1990): Referral letters and replies from orthopaedic departments: opportunities missed. BMJ 301, 470-3

Kassenärztliche Vereinigung Nordrhein (2006): Die 100 häufigsten ICD-10-Schlüssel und Kurztexte (nach Fachgruppen) 4. Quartal 2006.

http://www.kvno.de/importiert/100icd_06-4.pdf. (letzter Zugriff am 06.01.2008)

Kendrick D, Fielding K, Bentley E, Kerslake R, Miller P, Pringle M (2001): Radiography of the lumbar spine in primary care patients with low back pain: randomised controlled trial. Br Med J 322, 400-5

Knottnerus JA, ten Velden GH (2007): Dutch doctors and their patients--effects of health care reform in the Netherlands. N Engl J Med 357, 2424-6.

Krauth C, Grobe T, Hoopmann M Schwartz FW, Walter U: Kreuzschmerz: Krankheitskosten und Einsparpotentiale präventiver Interventionen. In : Hildebrandt J, Müller G, Pfingsten M: Lendenwirbelsäule. Ursachen, Diagnostik und Therapie der Rückenschmerzen. Urban \& Fischer, München 2005, 14-25

Lang E, Kastner S, Neundörfer B (2002): Verbesserung der ambulanten Versorgung von Patienten mit chronischen Rückenschmerzen. Schmerz 16, 22-33

McPhee SJ, Lo B, Saika GY, Meltzer R (1984): How good is communication between primary care physicians and subspecialty consultants? Arch Intern Med 144, 1265-8

Merten M (2008): Praxisgebühr: Verpuffter Steuerungseffekt http://www.aerztlichepraxis.de/artikel_politik_aktuell_medi_1199706280.htm (letzter Zugriff am 16.07.2008)

Müller G (2001): Diagnostik des Kreuzschmerzes. Wo liegen die Probleme? Schmerz $6,435-41$

Newton J, Eccles M, Hutchinson A (1992): Communication between general practitioners and consultants: what should their letters contain? Br Med J 304, 821-4

Pengel LH, Herbert R, Maher CG, Refshauge KM (2003): Acute low back pain: A systematic review of its prognosis. Br Med J 327, 323-27

Pfingsten M, Hildebrandt J (2001): Die Behandlung chronischer Kreuzschmerzen durch ein intensives Aktivierungskonzept (GRIP) - eine Bilanz von 10 Jahren. Anaesthesiol Intensivmed Notfallmed Schmerzther 36, 580-9.

Pfingsten M, Schöps P (2004): Chronische Rückenschmerzen: Vom Symptom zur Krankheit. Z Orthop Ihre Grenzgeb 142, 146-52

Raspe H (2008): Management of chronic low back pain in 2007-2008. Curr Opin Rheumatol 20(3), 276-81 
Raspe H, Kohlmann T (1993): Kreuzschmerzen -eine Epidemie unserer Tage? Dtsch Arztebl, 90, 2920-5

Rosemann T, Wensing M, Rueter G, Szecsenyi J (2006): Referral letters from general practice to consultants in Germany: If the GP is the initiator, patients' experiences are more positive. BMC Health Serv Res 6:5

Sachverständigenrat zur Begutachtung der Entwicklung im Gesundheitswesen (2001): Band. III: Über-, Unter- und Fehlversorgung, Nomos Verlag, Baden-Baden. http://www.svr-gesundheit.de/Gutachten/Gutacht01/Kurzf-de.pdf (letzter Zugriff am 06.01.2008)

Schmidt CO, Raspe H, Pfingsten M, Hasenbring M, Basler D HD, Eich W, Kohlmann $T$ (2007): Back pain in the german adult population. Spine 32, 2005-11

Von Korff M (1994): Studying the natural history of back pain. Spine 19(18 Suppl), 2041-2046

Waddell G (1982): An approach to backache. Br J Hosp Med 23, 187-219

Wetzel D, Himmel W, Heidenreich R (2005): Participation in a quality of care study and consequences for generalizability of general practice research. http://fampra.oxfordjournals.org/cgi/content/full/22/4/458 (letzter Zugriff am 07.01.2009)

Wey, Michael (2004): STANDPUNKT: Entbürokratisierung. Dtsch Arztebl 101(1-2): A-68 / B-60 / C-60 


\section{Abbildungsverzeichnis}

Seite

Abbildung 1: $\quad$ Rekrutierungsverlauf der teilnehmenden Hausarztpraxen $\quad 19$

Abbildung 2: $\quad$ Selbsteinschätzung der Hausärzte in Bezug auf ihre Angaben bei der Überweisung (Angaben in \%)

Abbildung 3: $\quad$ Angaben der Hausärzte auf dem Überweisungsschein (Angaben in \%)

Abbildung 4: $\quad$ Gründe für die Überweisung zum Orthopäden (Angaben in \%)

Abbildung 5: $\quad$ Angaben der Orthopäden im Befundbericht (Angaben in \%)

Abbildung 6: $\quad$ Zufriedenheit der Hausärzte mit dem orthopädischen Befundbericht (Angaben in \%) 
10. Tabellenverzeichnis

Seite

Tabelle 1: $\quad$ Warnhinweise auf komplizierte Kreuzschmerzen 


\section{Abkürzungsverzeichnis}

AOK Allgemeine Ortskrankenkasse

DEGAM Deutsche Gesellschaft für Allgemeinmedizin und Familienmedizin

EBM Einheitlicher Bewertungsmaßstab

EDV Elektronische Datenverarbeitung

HIV Humanes Immundefizienz-Virus

ICD-10 International Classification of Diseases

IGOST Interdisziplinäre Gesellschaft für orthopädische und unfallchirurgische Schmerztherapie e.V.

NSAR nichtsteroidale Antirheumatika

SAS Statistical Analysis System (Softwaresystem) 


\section{Anhang}

\subsection{Anlage 1: Fragebogen 1}

„Überweisung zum Orthopäden bei Kreuzschmerzen“

Praxiscode

\section{Vorgehen bei der Überweisung von Patienten zum Orthopäden}

Trifft völlig

zu

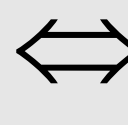

Trifft nicht zu

Ich gebe bei Überweisungen einen klaren

Arbeitsauftrag (Zielauftrag)

Ich mache dabei Angaben zu

$\begin{array}{llllll}\text { Schmerzdauer } & \square & \square & \square & \square & \square \\ \text { Schmerzcharakter } & \square & \square & \square & \square & \square \\ \text { Schmerzausstrahlung } & \square & \square & \square & \square & \square \\ \text { Kraftprüfung } & \square & \square & \square & \square & \square \\ \text { Beweglichkeit } & \square & \square & \square & \square & \square \\ \text { bisheriger Therapie } & \square & \square & \square & \square & \square \\ \text { Nebendiagnosen (Komorbidität) } & \square & \square & \square & \square & \square\end{array}$

Wird nur einmal ausgefüllt! 


\subsection{Anlage 2: Fragebogen 2}

„Beurteilung der orthopädischen Befundberichte durch die Hausärzte“

Praxiscode Patienten -Nr. Alter Geschlecht

\section{Beurteilung des orthopädischen Befundberichtes}

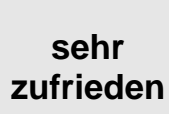

weiß

nicht/

trifft

nicht zu

\begin{tabular}{|c|c|c|c|c|c|c|}
\hline Struktur & $\square$ & $\square$ & $\square$ & $\square$ & $\square$ & $\square$ \\
\hline Diagnose & $\square$ & $\square$ & $\square$ & $\square$ & $\square$ & $\square$ \\
\hline Befunddokumentation & $\square$ & $\square$ & $\square$ & $\square$ & $\square$ & $\square$ \\
\hline Angaben zur durchgeführten Diagnostik & $\square$ & $\square$ & $\square$ & $\square$ & $\square$ & $\square$ \\
\hline Angaben zur durchgeführten Therapie & $\square$ & $\square$ & $\square$ & $\square$ & $\square$ & $\square$ \\
\hline Angaben zur weiteren Therapie & $\square$ & $\square$ & $\square$ & $\square$ & $\square$ & $\square$ \\
\hline Lesbarkeit & $\square$ & $\square$ & $\square$ & $\square$ & $\square$ & $\square$ \\
\hline Gesamtbeurteilung & $\square$ & $\square$ & $\square$ & $\square$ & $\square$ & $\square$ \\
\hline Initiative für die Überweisung & & & ifft z & & trifft nicht zu & $\begin{array}{l}\text { weiß } \\
\text { nicht }\end{array}$ \\
\hline Initiative für die Überweisung kam von mir & & & $\square$ & & $\square$ & $\square$ \\
\hline Initiative für die Überweisung kam vom Patienten & & & $\square$ & & $\square$ & $\square$ \\
\hline
\end{tabular}

$\begin{array}{lccc}\text { Gründe für die Überweisung } & \text { trifft zu } & \text { trifft nicht zu } & \begin{array}{l}\text { nicht } \\ \text { bekannt }\end{array} \\ \text { Wunsch meinerseits nach Diagnoseüberprüfung } & & \square & \square \\ \text { Wunsch des Patienten nach Diagnoseüberprüfung } & \square & \square & \square \\ \text { Patient soll erkennen, dass die diagnostischen und } & \square & \square & \square \\ \text { therapeutischen Möglichkeiten ausgeschöpft sind } & \square & \square & \square \\ \text { Patient hat länger als vier Wochen Beschwerden } & \square & \square & \square \\ \text { Patient hat Chronifizierungspotential } & \square & \square \\ \text { Patient ist psychosozial belastet } & \square & \square & \square \\ \text { Patient ist unzufrieden mit bisheriger Behandlung } & \square & \square & \square \\ \text { Patient leidet unter anhaltenden Belastungen im privaten } & \square & \square \\ \text { Alltag } & \square & \square & \square \\ \text { Patient leidet unter einer belastenden Arbeitssituation } & \square & \square & \square \\ \text { Patient hat Rentenwunsch } & \square & \square & \square \\ \text { Überweisung aus Budgetgründen } & \square & \square & \square \\ \text { Überweisung aus forensischen Gründen } & \square & \square & \square \\ \text { Ich möchte mich vom Patienten entlasten } & \square & \square & \square \\ \text { Ich konnte dem Patienten nicht mehr helfen } & & \square\end{array}$


12.3 Anlage 3: Procedure Normal

data $x$;

do $X=1$ to 120 ; output; end; run;

data $\mathrm{xx}$;

set $x$;

zuf $=$ normal $(0)$;

if zuf $>0.058$ then output;

run;

options linesize $=65$ pagesize $=600$;

proc print data $=\mathrm{xx}$;

var X; run;

Das SAS System 14

13:12 Tuesday, January 9, 2007

Beob. X

12

24

37

48

510

612

718

819

921

1024

1125

1226

1328

1429

1530

1635

1736

1837

1938

2039

2140

2245

2346

2448

2549

2650

2751

2852

2953

3054

3155

3256

3358 
3461

3562

3664

3767

3868

3970

4072

4174

4276

4379

4480

4581

4686

4787

4888

4990

5092

5194

5295

5397

54100

55102

56104

57108

58111

59113

60115

61117

62119 


\subsection{Anlage 4: Erstdokumentation Rückenschmerzen}

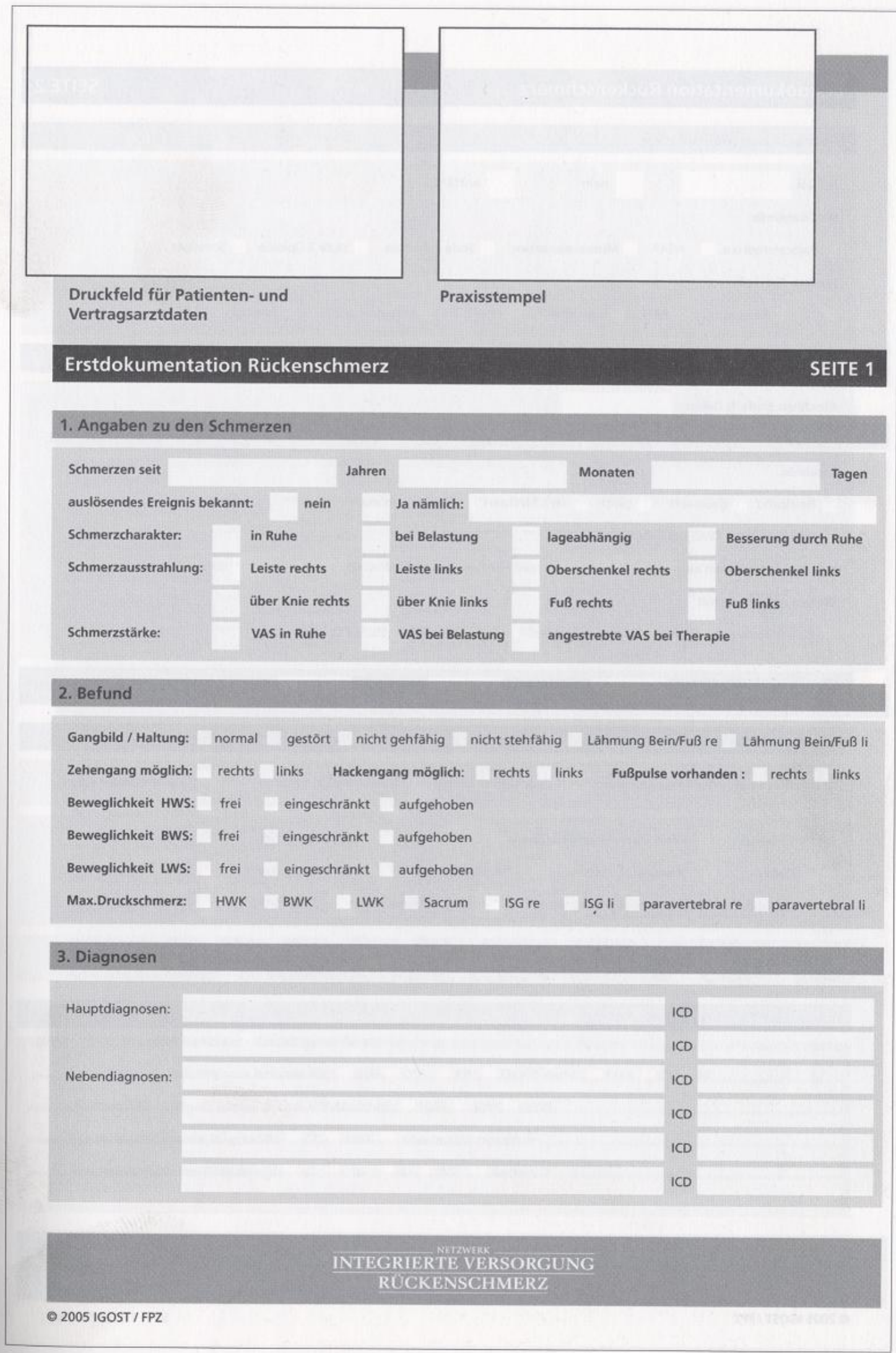

Abb. 3.4a: Erstdokumentation 


\section{Veranlasste Maßnahmen}

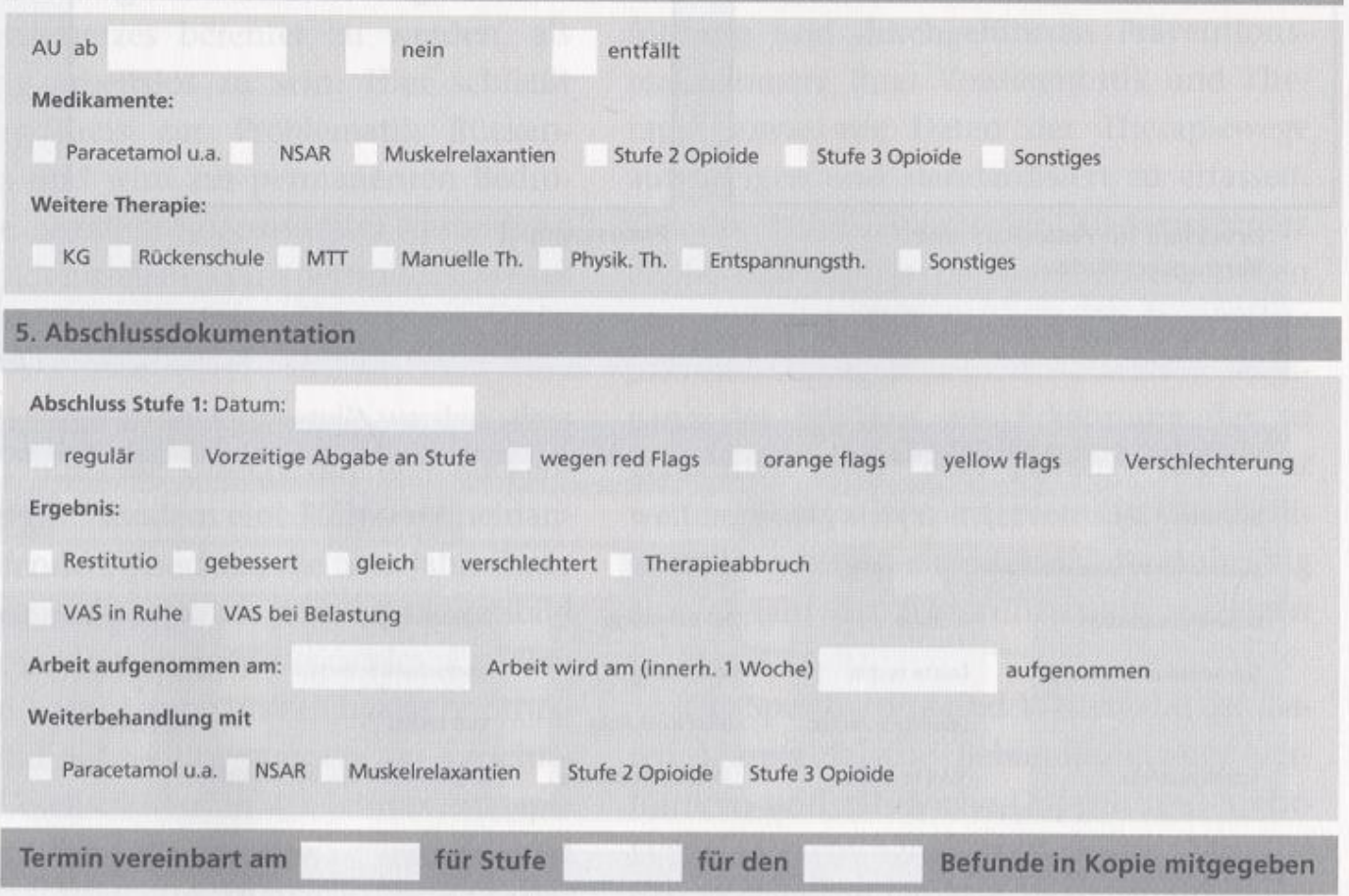

\section{Red/Orange/Yellow Flags}

\begin{tabular}{|c|c|c|c|c|}
\hline \multicolumn{2}{|c|}{ RED FLAGS > sofort ins Krankenhaus: } & Cauda-Syndrom & Querschnittssymptome & V.a. spinalen Abszess \\
\hline \multirow{2}{*}{$\begin{array}{l}\text { ORANGE FLAGS } \\
\text { Fieber }\end{array}$} & \multicolumn{2}{|c|}{ Sofort Öberweisung für Ebene } & Gewichtsverlust & \\
\hline & Immunsuppression & Tumoranamnese & Gefäßerkrankung & HIV \\
\hline V.a. abdom & le / extraspinale Erkrankung & V.a. Rheuma & Z.n. WS-Op. & Osteoporose \\
\hline
\end{tabular}

\begin{tabular}{|c|c|c|}
\hline YELLOW FLAGS & Sofort Überweisung Ebene 2 & V.a. psychiatrische Komorbiditàt \\
\hline
\end{tabular}

\section{Sonstige Bemerkungen}

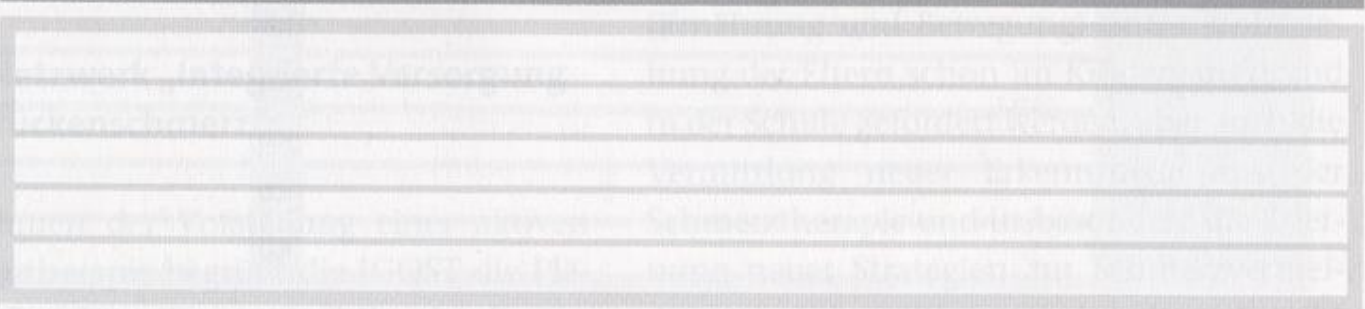

\section{INTEGRIERTIE VERSORGUNG}

RUCKENSCHMERZ

\section{IGOST / FPZ}

ortsetzung Abb. 3.4a: Erstdokumentation 


\subsection{Anlage 5: Votum Ethikkommission}

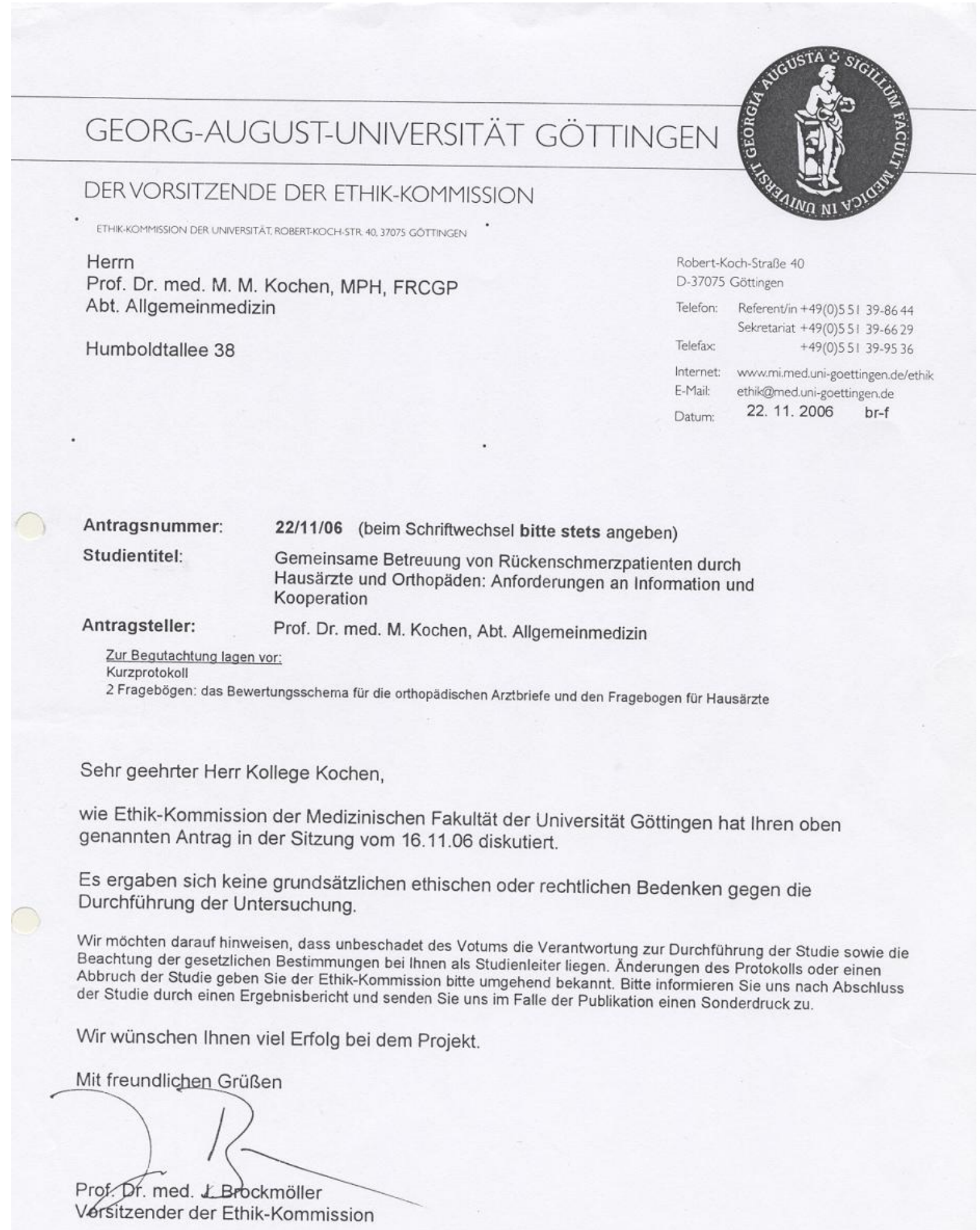




\subsection{Anlage 6: Patientenbegleitdokumentation AOK}

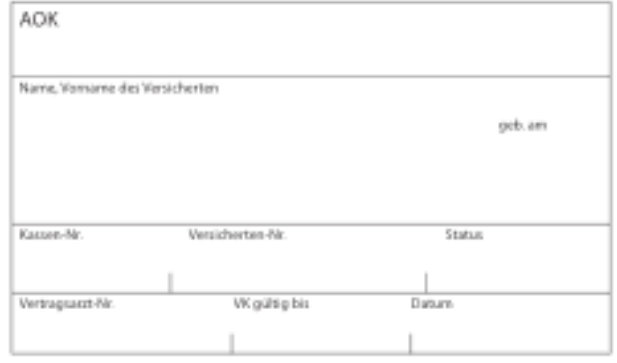

\section{Patienten- begleitdokumentation}

Integrationsversorgung Rückenschmerzen

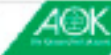

80 DEUTSCMFA

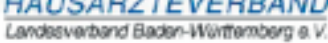

\section{Anamnese:}

I Rückenschmerz $\square$ akut seit:

D chronisch seit:

I Behandlungsbeginn: Arbeitsunfähig seit:

I Voroperation an Brust- oder Lendenwirbelsäule (s. Arztbrief in Anlage) D Ja Nein

I Akut stationäre Behandlung in den letzten 12 Monaten wegen Rückenschmerzen (s. Arztbrief in Anlage) D Ja $\square$ Nein

I Stat./amb. Reha in den letzten 12 Monaten wegen Rückenschmerzen D Ja Nein

I Psychiatr/psychotherap. Behandlung im Zusammenhang mit Rückenschmerzen Da Nein

I Teilnahme am AOK-Rückenprogramm
D Ja
D Nein

I berufliche/familiäre Besonderheiten:

\section{Befunde:}

I Größe: Gewicht:

I pathologische neurologische/andere Befunde:

\section{Bisherige Maßnahmen:}

• Analgetika:

$\square$ NSAR:

Z Zentral wirksame:

Muskelrelaxantien:

口 Physikalische Therapie:

口 Heilmittel:

Jonstige:

Verlauf:

Desserung:

eine Veränderung:

$\square$ Verschlimmerung:

Fragestellung: 


\section{Danksagung}

Für die freundliche Überlassung des Themas der Dissertation danke ich Herrn Prof. Dr. M. M. Kochen, MPH, FRCGP.

Herrn PD Dr. J.F. Chenot, MPH,

danke ich für die sehr engagierte und kontinuierliche Betreuung meiner Arbeit.

Herrn Prof. Dr. disc. pol. Wolfgang Himmel und den Mitarbeitern der Abteilung Allgemeinmedizin danke ich für ihre Kritik und Anregungen.

Mein besonderer Dank gilt den Ärztinnen, Ärzten und medizinischen Fachangestellten, die sich an dieser Studie beteiligt haben. 


\section{Curriculum vitae}

Am 31. März 1982 wurde ich als Tochter von Renate Jürgens-Pieper, geb. Mester, und Wilhelm Pieper in Braunschweig geboren.

Von 1988-1992 besuchte ich die Grundschule in Groß Schwülper. 1992 wechselte ich auf die Integrierte Gesamtschule Franzsches Feld in Braunschweig, wo ich im Jahre 2001 die Allgemeine Hochschulreife erhielt.

Im Sommersemester 2002 begann ich mein Studium der Humanmedizin an der Georg-August-Universität in Göttingen. Mein Krankenpflegepraktikum leistete ich 2003 in einem Entwicklungshilfe-Projekt in Kalkutta (Indien) ab.

Die ärztliche Vorprüfung (Physikum) bestand ich im Frühjahr 2004.

Famulaturen unternahm ich in Anästhesie in Westerstede, in der Gynäkologie und Geburtshilfe in Berlin-Lichtenberg, in Tropenmedizin in Same (Tansania), in Neurologie in Braunschweig und in Dermatologie in Göttingen.

Im Wintersemester 2005/2006 studierte ich im Rahmen eines ERASMUSStipendiums an der „Universidad de Salamanca“ in Spanien.

Vom August 2007 bis Juli 2008 absolvierte ich mein Praktisches Jahr in den Fächern Innere Medizin in Jersey (Großbritannien) und Göttingen, in Chirurgie als Unterassistentin im Kantonsspital Zug (Schweiz) und in der Pädiatrie in Hildesheim.

Die Zweite Ärztliche Prüfung legte ich im Oktober und November 2008 ab.

Zurzeit arbeite ich als Assistenzärztin in der Kinder- und Jugendpsychiatrie in Landshut. 